\title{
Photo-absorption and electron collection of field- assisted GaN nanohole array photocathode
}

Lei Liu ( $\square$ liu1133_cn@sina.com.cn )

njust https://orcid.org/0000-0002-4966-5914

\section{Feifei Lu}

Nanjing University of Science and Technology

Jian Tian

Nanjing University of Science and Technology

Xingyue Zhangyang

Nanjing University of Science and Technology

\section{Research Article}

Keywords: GaN, nanohole array, photocathode, electron collection, light absorption

Posted Date: February 15th, 2021

DOI: https://doi.org/10.21203/rs.3.rs-159096/v1

License: (c) (1) This work is licensed under a Creative Commons Attribution 4.0 International License.

Read Full License

Version of Record: A version of this preprint was published at Journal of Materials Science: Materials in Electronics on April 15th, 2021. See the published version at https://doi.org/10.1007/s10854-021-058947. 


\title{
Photo-absorption and electron collection of field-assisted GaN nanohole array photocathode
}

\author{
Lei Liu*, Feifei Lu, Jian Tian, Xingyue Zhangyang \\ Department of optoelectronic technology, School of Electronic and Optical \\ Engineering, Nanjing University of Science and Technology, Nanjing 210094, China \\ Corresponding author: Lei Liu; E-mail: liu1133_cn@sina.com.cn
}

\begin{abstract}
The light absorption and photo-generation rate under different periods, filling factors (FF), hole depth and inclination angles are studied. The NHA exhibits a larger light absorption compared with the planar film, which is about $99.99973 \%$. Based on the three-dimensional continuity equation, the quantum efficiency $(\mathrm{QE})$ and collection efficiency (CE) of the field-assisted GaN NHA and the graded compositional AlGaN NHA are calculated. The QE and CE of the GaN NHA with a period of $200 \mathrm{~nm}$, a filling factor of 0.05 , an inclined angle of $10^{\circ}$, and a field intensity of $2 \mathrm{~V} / \mu \mathrm{m}$ are $62.7 \%$ and $62.6 \%$, respectively. In addition, the graded compositional $\mathrm{AlGaN}$ structure has a more improved effect on the vertical NHA. Compared with the uniform GaN NHA, the electron collection of AlGaN NHA ratio is increased by 2.4 times. The design principles proposed in this work provide guidance to reasonable parameters for the application of NHA photocathodes.
\end{abstract}

Key words: GaN; nanohole array; photocathode; electron collection; light absorption.

\section{Introduction}

GaN-based photocathode devices have the advantages of large band gap, high breakdown voltage, high quantum efficiency $(\mathrm{QE})$ and stable chemical properties $[1,2]$. It is an important material for the development of ultraviolet photocathodes. With the development of nanotechnology, low-dimensional structures have broad development prospects in optoelectronic applications [3-5]. Some new types of nanostructures can 
make full use of the energy in the sun to turn into the electrical energy we need in our daily lives, for example, nanowires [6], nanocones [7], nano-pyramid [8], moth-eye [9], nanohole [10], even nano-cone-hole [11] and so on. Chen et al. used polystyrene nanosphere lithography and reactive ion etching technology to fabricate silicon nanohole arrays (NHA) in a large area process, which increased the power conversion efficiency by $19 \%$ [12]. Hong et al. used the finite element method to study the inclined Si NHA structure, and achieved the highest ultimate efficiency of $32.9 \%$ [13]. By selecting the best parameters of the inclined nanohole, Deng et al. can achieve a shortcircuit photocurrent density of $31.2 \mathrm{~mA} / \mathrm{cm}^{2}$, which is $10.25 \%$ higher than the best vertical nanohole system and $105.26 \%$ higher than bare Si with a thickness of $2330 \mathrm{~nm}$ [14]. The open circuit voltage of Si nanohole solar cell with p-n junction through P diffusion by Peng et al. is $566.6 \mathrm{mV}$, the short-circuit current density is $32.2 \mathrm{~mA} / \mathrm{cm}^{2}$, and the power conversion efficiency is as high as 9.51\% [15]. Zhang et al. proposed a dual-diameter nanohole photovoltaic system that uses a top layer (bottom) with large (small) nanoholes to improve the absorption of short-wavelength (long-wavelength) solar incidents, and the photocurrent density is expected to reach $27.93 \mathrm{~mA} / \mathrm{cm}^{2}$, which is $17.39 \%$ higher than the best single hole, achieving a light conversion efficiency of $13.72 \%$ [16]. Nano-materials with different structures proposed in recent years have comprehensive anti-reflection properties and optical absorption enhancement properties [17-19], which provide new possibilities for conventional optoelectronic devices. In view of the superiority of GaN materials in ultraviolet absorption and the high absorption characteristics of inclined NHA, GaN inclined NHA have broad application prospects in the field of photocathode, which can be used as ideal for ultraviolet photocathode emission layers material.

For photocathodes, improving QE and the effective utilization of electrons has always been an important research content. Zou et al. introduced a graded doping structure in the photocathode emission layer to generate an electric field, which helps electrons escape [20]. Fu and Yang et al. also introduced exponential doping and graded compositional structure to enhance QE $[21,22]$. In previous studies, we separately discussed the QE of photocathodes with uniform doping and exponential doping GaN 
nanowire arrays. The structure that can introduce a built-in electric field can indeed increase the carrier migration [23,24]. Except the internal variable doping structure, field assist is another way to improve the efficiency of electron utilization, and the application of an external electric field also help electron transfer [25,26]. Therefore, it is necessary to explore the $\mathrm{QE}$ and electron collection ratio of the field-assisted $\mathrm{GaN}$ NHA photocathode.

In this work, based on the finite-difference time-domain (FDTD) method, a model of GaN and graded compositional AlGaN inclined NHA cathode was established to obtain the photogeneration rate, and the influence of period, filling factor (FF), hole depth and inclined angle to photo-absorption were analyzed. Then, the threedimensional continuity equation is used to simulate carrier concentration distribution and QE. Finally, the collection efficiency (CE) of inclined NHA cathode under the external electric field are calculated according to the initial energy and angular distribution of the electrons. This work can provide theoretical reference for the application of GaN NHA photocathode.

2. Theoretical model

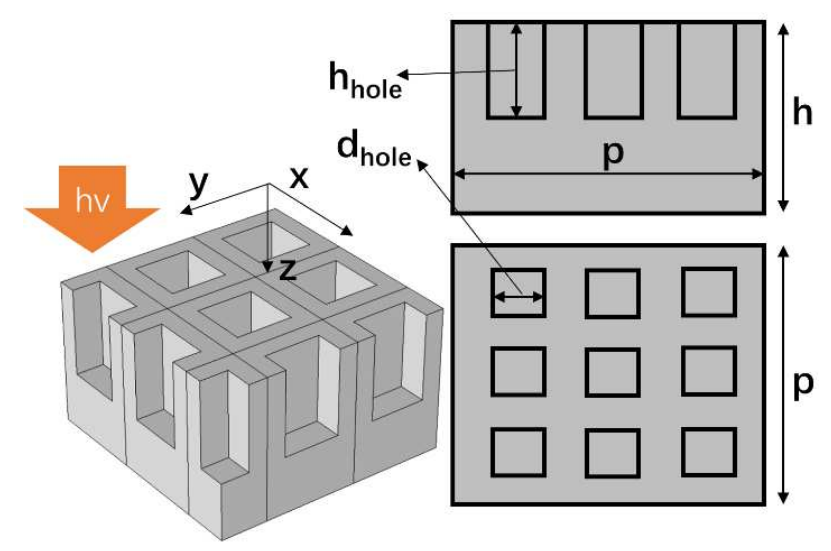

Figure 1. Structural model of GaN nanohole array.

The left side of Fig. 1 shows a simplified diagram of the GaN NHA structure, and the right side shows the side view and top view of the square NHA. The period $p$, the depth $h_{\text {hole }}$ and the diameter $d_{\text {hole }}$ of the hole are set as basic structural parameters. The ratio of the area of the GaN material to the area of all cell square on the horizontal section is defined as the filling factor [27], then the filling factor of the square NHA is $\mathrm{ff}=1-\frac{N \times d_{\text {hole }}{ }^{2}}{p^{2}}$, where $\mathrm{N}$ is the number of cells in the $\mathrm{p}^{2}$ area. The total depth $h$ of the 
entire NHA is set to $2000 \mathrm{~nm}$. The transmittance monitor is set at the bottom of the entire nanohole structure, and the reflectance monitor is set at a distance of $1000 \mathrm{~nm}$ from the top of the nanohole. The three-dimensional electric field monitor is placed in the entire simulation domain to accurately simulate the internal electric field of the material after the fine division of a uniform grid of $2 \mathrm{~nm} \times 2 \mathrm{~nm} \times 2 \mathrm{~nm}$. The plane wave is placed 500nm away from the top of the array as the incident light source. Perfectly matched layer boundary conditions are applied to the top and bottom respectively, and the boundary in the in-plane direction uses periodic boundaries to simulate the array structure. Unpolarized light absorption is calculated by averaging the average of TM and TE polarization. Calculate the absorption of inclined GaN NHA by reflectance $R(\lambda)$ and transmittance $\mathrm{T}(\lambda)[27]$ :

$$
A(\lambda)=1-R(\lambda)-T(\lambda)
$$

The electric field can be used to calculate the photo-generation rate of electron-hole pairs in the simulation area:

$$
G(x, y, z, \lambda)=\frac{\pi \varepsilon^{\prime \prime} E(x, y, z, \lambda)^{2}}{\hbar}
$$

where $\hbar$ and $\varepsilon$ " are Planck's constant and the imaginary parts of dielectric constant, respectively. The three-dimensional carrier continuity equation of the GaN NHA photocathode can be expressed [28]:

$$
D_{n} \nabla^{2} n(x, y, z, \lambda)-\frac{n(x, y, z, \lambda)}{\tau}+G(x, y, z, \lambda)=0
$$

where $D_{n}$ is the diffusion coefficient, $\tau$ represents the lifetime of the carrier, and $n(x, y, z, \lambda)$ represents the carrier concentration of a certain wavelength. The analytical solution of the carrier concentration is difficult to derive or is too complicated, so the finite difference method is used to realize the carrier concentration of each very small unit in the material. The bottom surface and the top surface are respectively set as ohmic contact and charge emission boundary conditions. All electrons reaching the cathode surface will undergo emission or recombination, and the electron density should be very low, ideally 0 at the surface boundary. Assign the emission boundary equation to obtain the emission electron flow $J[29]$ : 


$$
\left\{\begin{array}{c}
J_{\text {top }}(\lambda)=\left.q P D_{n} \nabla n(x, y, z, \lambda)\right|_{\text {top surface }} \\
J_{\text {sides }}(\lambda)=\left.q P D_{n} \nabla n(x, y, z, \lambda)\right|_{\text {sides }} \\
J_{\text {underside }}(\lambda)=\left.q P D_{n} \nabla n(x, y, z, \lambda)\right|_{\text {underside }}
\end{array}\right.
$$

where $q$ is the basic charge and $P$ is the escape probability of electrons emitted into the vacuum. Then the $\mathrm{QE}$ after emission can be calculated as:

$$
\mathrm{QE}=\frac{J_{\text {top }}+J_{\text {sides }}+J_{\text {underside }}}{I_{0} S}
$$

where $I_{0}$ is the intensity of the incident light, and $S$ is the surface area of the light incident surface.

For graded compositional AlGaN NHA, the $\mathrm{Al}$ composition must be designed to show a gradient downward trend from the substrate side $(\mathrm{z}=0)$ to the top of the nanowire $(\mathrm{z}=\mathrm{H})$ along the growth direction ( $\mathrm{z}$ axis). Only in this way can the built-in electric field pointing from the top surface to the substrate side be introduced [30]. The bottom of the NHA is designed with $\mathrm{Al}_{0.6} \mathrm{Ga}_{0.4} \mathrm{~N}$ material, and the top is still $\mathrm{GaN}$ material to facilitate electron emission. The refractive index of $\mathrm{Al}_{0.6} \mathrm{Ga}_{0.4} \mathrm{~N}$ material comes from Liu's report [31]. After the built-in electric field is introduced, the three-dimensional carrier continuity equation is rewritten as [22]:

$$
D_{n} \nabla^{2} n(x, y, z, \lambda)-\mu E(z) \frac{\partial n(x, y, z, \lambda)}{d z}-\frac{n(x, y, z, \lambda)}{\tau}+G(x, y, z, \lambda)=0
$$

where $\mu$ is the electron mobility and $E(z)$ represents the total electric field at point z. The conduction band offset between $\mathrm{Al}$ constituent materials can be estimated as [32]:

$$
\Delta E_{c n}=\left|\left(x_{n}^{2}-x_{n-1}^{2}\right)+0.8 \cdot\left(x_{n}-x_{n-1}\right)\right| \mathrm{eV}
$$

where $x_{n}$ and $x_{n-1}$ respectively represent the $\mathrm{Al}$ content of two adjacent materials. Then the built-in electric field can be expressed as:

$$
E_{\text {in }}(z)=\Delta E_{c n} / H_{i}
$$

where $\mathrm{H}_{\mathrm{i}}$ represents the thickness of each sublayer, and $E_{\text {in }}(z)$ is the built-in electric field. The band gaps of $\mathrm{Al}_{0.6} \mathrm{Ga}_{0.4} \mathrm{~N}$ and $\mathrm{GaN}$ materials are estimated to be $3.632 \mathrm{eV}$ and $3.42 \mathrm{eV}$, respectively [33]. Therefore, the total electric field of the field-assisted AlGaN NHA can be expressed as:

$$
E_{\text {ext }}(z)+E_{\text {in }}(z)=E(z)
$$

The process of solving QE is the same as that of inclined GaN NWA. 

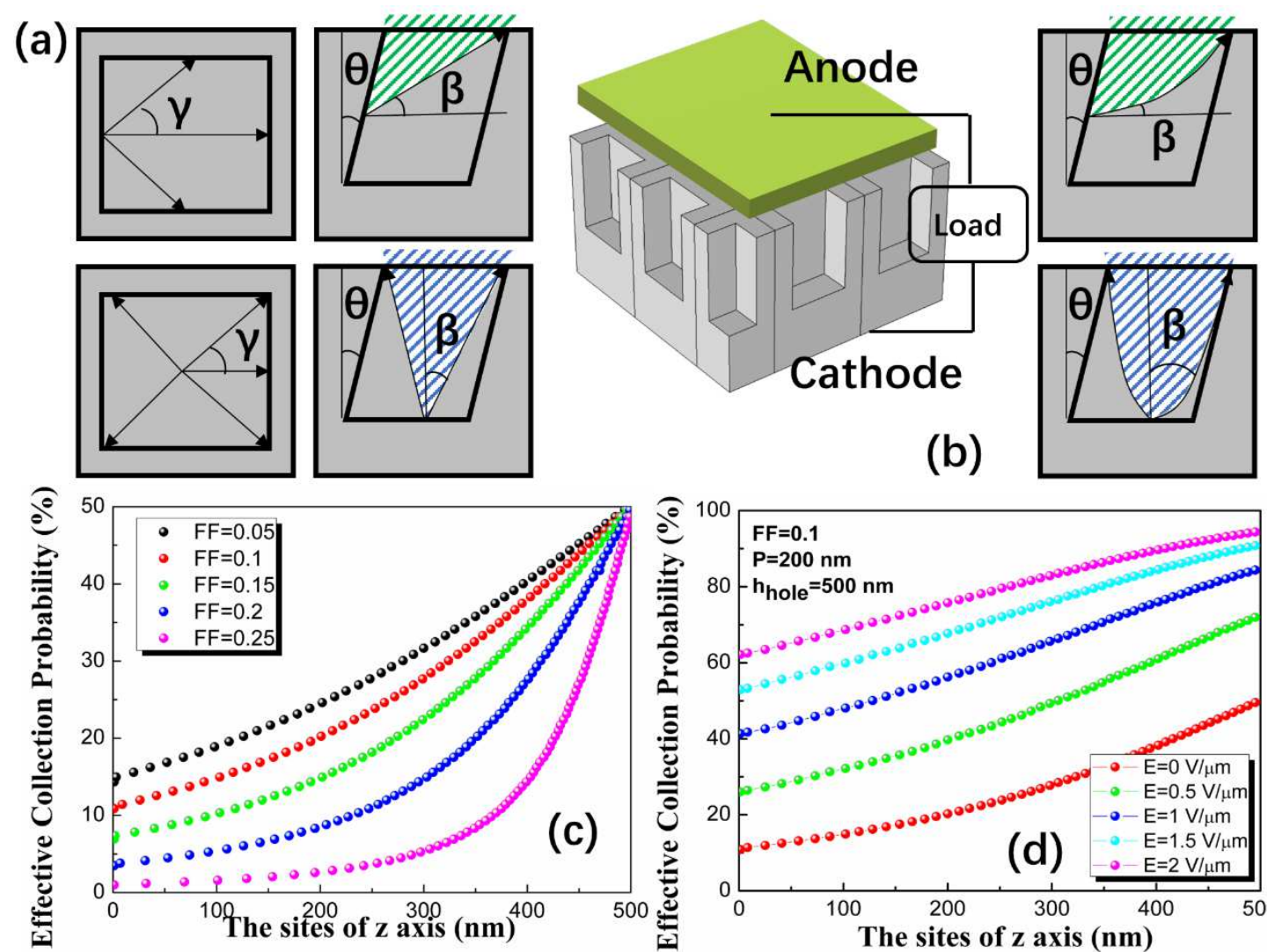

(b)

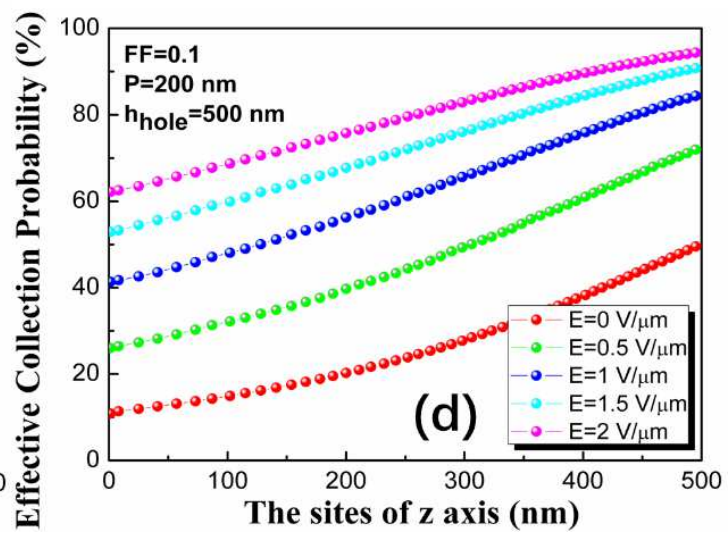

Figure 2. (a) The range where electrons can be collected from the inclined GaN NHA under no external electric field. (b) Left: A schematic diagram of the device model of the field-assisted GaN NHA. Right: The range where electrons can be collected from the inclined GaN NHA under an external electric field. (c) The dependence of the effective collection probability of electrons emitted from the side of the GaN NHA on the filling factor when there is no external electric field. (d) The dependence of the effective collection probability of electrons emitted from the side of the GaN NHA on the field intensity.

In the effective electron collection process of the three-dimensional model of the NHA with a inclined angle of $\theta$, the collection side is set on the top of the NHA to collect electrons. However, the electrons emitted from the side of the nanohole are easily "secondarily absorbed" by the opposite side of the nanohole, and cannot reach the collection end on the top of the cathode. Fig. 2(a) shows the trajectory of the emitted electrons in the x-y plane and the x-z plane. The angles of electrons randomly emitted into the vacuum are divided into $\gamma$ and $\beta$. The initial angle $\gamma, \beta$ and energy $\varepsilon$ distribution satisfy the cosine law and Henke model respectively [34, 35]:

$$
\left\{\begin{array}{l}
N(\gamma)=\cos \gamma \\
N(\beta)=\cos \beta \\
N(\varepsilon)=\frac{a \varepsilon}{(\varepsilon+b)^{4}}
\end{array}\right.
$$

Photogenerated carriers escape from the top, side and bottom surfaces of the NHA, 
and electrons emitted from the top surface can be collected by the collection side regardless of the angle they exit. However, the electrons on the side and bottom can only be collected in the green and blue regions respectively, as shown in Fig. 2(a). Then, the conditions under which the emitted electrons from the side are collected can be summarized as:

$$
P_{C}=\frac{\int_{\gamma=-90^{\circ}}^{\gamma=90^{\circ}} \int_{\theta+\beta<\arctan \frac{\left(h_{h o l e}-z\right) \cos \theta}{d} \int_{\text {hole }}}^{\beta=90^{\circ}-\theta} \int_{\varepsilon=0}^{\varepsilon=1} N(\varepsilon) N(\beta) N(\gamma) d \varepsilon d \beta d \gamma}{\int_{\gamma=-90^{\circ}}^{\gamma=90^{\circ}} \int_{\beta=90^{\circ}+\theta}^{\beta=90^{\circ}-\theta} \int_{\varepsilon=0}^{\varepsilon=1} N(\varepsilon) N(\beta) N(\gamma) d \varepsilon d \beta d \gamma}
$$

When the exit elevation angle is $0 \leq \beta \leq 90^{\circ}$, the conditions under which the emitted electrons on the bottom surface are collected can be summarized as:

$$
P_{d}=\frac{\int_{\gamma=-180^{\circ}}^{\gamma=180^{\circ}} \int_{\beta=0^{\circ}}^{\frac{|x|}{\tan \beta}>H \cos \theta} \int_{\varepsilon=0}^{\varepsilon=1} N(\varepsilon) N(\beta) N(\gamma) d \varepsilon d \beta d \gamma}{\int_{\gamma=-180^{\circ}}^{\gamma=180^{\circ}} \int_{\beta=0^{\circ}}^{\beta=90^{\circ}} \int_{\varepsilon=0}^{\mathcal{E}=1} N(\varepsilon) N(\beta) N(\gamma) d \varepsilon d \beta d \gamma}
$$

When the exit elevation angle is $-90^{\circ} \leq \beta<0^{\circ}$, the conditions under which the emitted electrons on the bottom surface are collected can be summarized as:

$$
P_{d}=\frac{\int_{\gamma=-180^{\circ}}^{\gamma=180^{\circ}} \frac{\int_{d_{h o l e l}-|x|}^{\beta=0^{\circ}}>H \cos \theta}{\tan \beta} \int_{\varepsilon=0}^{\varepsilon=1} N(\varepsilon) N(\beta) N(\gamma) d \varepsilon d \beta d \gamma}{\int_{\gamma=-180^{\circ}}^{\gamma=180^{\circ}} \int_{\beta=-90^{\circ}}^{\beta=0^{\circ}} \int_{\mathcal{E}=0}^{\varepsilon=1} N(\varepsilon) N(\beta) N(\gamma) d \varepsilon d \beta d \gamma}
$$

However, as shown in Fig. 2(b), when there is an external electric field, the pulling of the electric field force can make the electrons form a curved track in the nanohole, so that the electrons emitted from the side and bottom surface can be collected in a wider range. Then, the side collection probability is changed to:

$$
P_{C}=\frac{\int_{\gamma=-90^{\circ}}^{\gamma=90^{\circ}} \int_{L \tan (\beta+\theta)+\frac{L^{2} E_{\operatorname{ext} t}}{\beta=9 \cos ^{2} \beta} \geq(H-z) \cos \theta}^{\int_{\varepsilon=0}^{\varepsilon=1} N(\varepsilon) N(\beta) N(\gamma) d \varepsilon d \beta d \gamma}}{\int_{\gamma=-90^{\circ}}^{\gamma=90^{\circ}} \int_{\beta=90^{\circ}+\theta}^{\beta=90^{\circ}-\theta} \int_{\varepsilon=0}^{\varepsilon=1} N(\varepsilon) N(\beta) N(\gamma) d \varepsilon d \beta d \gamma}
$$

When the exit elevation angle is $0 \leq \beta \leq 90^{\circ}$, the conditions on the bottom surface are collected can be rewritten as:

$$
P_{d}=\frac{\int_{\gamma=-180^{\circ}}^{\gamma=180^{\circ}} \int_{\beta=0^{\circ}}^{\frac{|x|}{\tan \beta}+\frac{|x|^{2} E_{\text {ext }}}{4 \varepsilon \sin ^{2} \beta}>H \cos \theta} \int_{\varepsilon=0}^{\varepsilon=1} N(\varepsilon) N(\beta) N(\gamma) d \varepsilon d \beta d \gamma}{\int_{\gamma=-180^{\circ}}^{\gamma=180^{\circ}} \int_{\beta=0^{\circ}}^{\beta=90^{\circ}} \int_{\varepsilon=0}^{\varepsilon=1} N(\varepsilon) N(\beta) N(\gamma) d \varepsilon d \beta d \gamma}
$$

When the exit elevation angle is $-90^{\circ} \leq \beta<0^{\circ}$, the conditions on the bottom surface are collected can be rewritten as:

$$
P_{d}=\frac{\int_{\gamma=-180^{\circ}}^{\gamma=180^{\circ}} \frac{\int_{d_{h o l e}-|x|}^{\beta=0^{\circ}}+\frac{\left(d_{h o l e}-|x|\right)^{2} E_{\text {ext }}}{4 t a n \beta}>H \cos \theta}{\int_{\mathcal{E}=0}^{\varepsilon=1} N(\varepsilon) N(\beta) N(\gamma) d \varepsilon d \beta d \gamma}}{\int_{\gamma=-180^{\circ}}^{\gamma=180^{\circ}} \int_{\beta=-90^{\circ}}^{\beta=0^{\circ} \beta} \int_{\varepsilon=0}^{\varepsilon=1} N(\varepsilon) N(\beta) N(\gamma) d \varepsilon d \beta d \gamma}
$$

Therefore, the collection probability of the inclined GaN NHA photocathode can be 
expressed as:

$$
C E=\frac{P D_{n}}{I_{0} S}\left[J_{\text {top }}(\lambda)+P_{c} J_{\text {sides }}(\lambda)+P_{d} J_{\text {underside }}(\lambda)\right]
$$

Fig. 2(c) shows the effective collection probability $P_{c}$ of electrons emitted from sides of NHA with different filling factors $(\mathrm{FF})$ without an external electric field. A small FF means a larger diameter of the nanohole. In the NHA with a larger diameter, side electrons have a greater chance of being collected by the collecting side, especially with the electrons emitted at a higher position on the side of the nanohole. The diameter of the nanohole is equivalent to the pitch in the nanowire array. The larger the diameter of the hole in the array, the longer the electrons will fly in the nanohole. With the introduction of an external electric field, it is found that the probability of effective electron collection on the side surface has been substantially improved. The electrons emitted from the side surface close to the top surface are even completely collected, as shown in Fig. 2(d). The side-emitting electrons close to the bottom already have a collection probability of more than $60 \%$ at a field intensity of $2 \mathrm{~V} / \mu \mathrm{m}$. Meanwhile, as the field intensity continues to increase, the gap in the probability of effective electron collection gradually decreases.

3. Results and discussion

3.1 Photo-absorption enhancement of GaN nanohole array
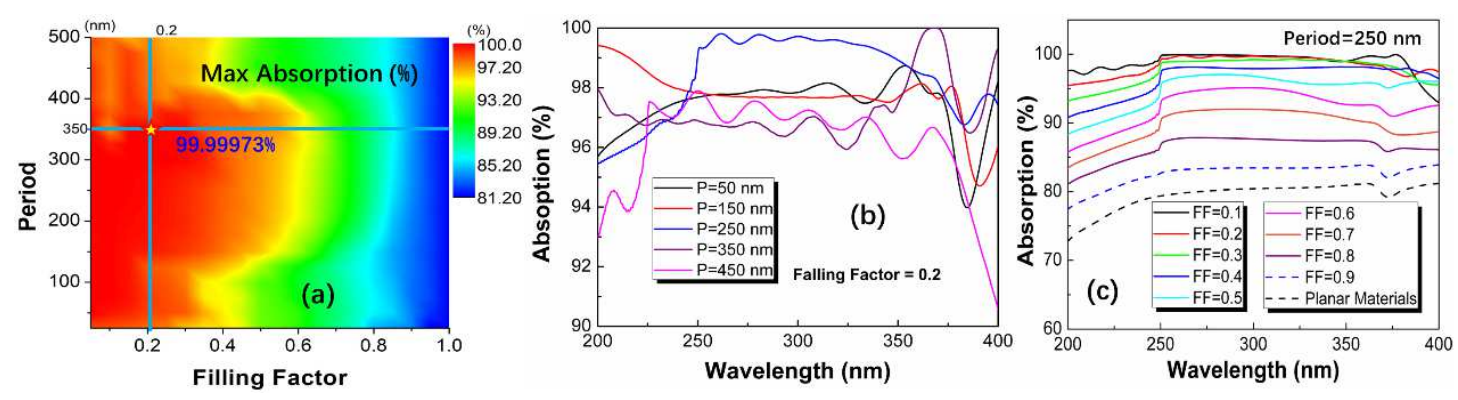

Figure 3. (a) Photo-absorption of a square vertical NHA as a function of period and filling factor. (b) When the filling factor is 0.2 , the dependence of the absorption spectrum of the vertical NHA on the period. (c) When the period is $250 \mathrm{~nm}$, the dependence of the absorption spectrum of the vertical NHA on the filling factor.

Fig. 3(a) shows the dependence of the maximum light absorption distribution on the filling factor and period of the vertical GaN NHA with a nanohole depth of $500 \mathrm{~nm}$. According to Fang's report [36], the optimal light absorption period can basically be 
determined near the operating wavelength, so we limit the period to $50 \mathrm{~nm}-500 \mathrm{~nm}$ near the operating wavelength range of the GaN photocathode, with the interval of $50 \mathrm{~nm}$. The variation range of the filling factor is $0-1$, and the interval is 0.05 . It is found that the best period does occur between the ultraviolet wavelengths of $150 \mathrm{~nm}-400 \mathrm{~nm}$. This phenomenon can be explained by the theory of wave optics. When $p$ is small, the wavelength is much larger than the typical characteristic size of the nanohole, and longwavelength light will easily pass through the nanohole structure, resulting in high transmittance. When the size of the nanohole is equivalent to the ultraviolet wavelength, it will cause strong scattering, which greatly extends the effective light passage length of light [13]. Lower filling factor means less material consumption. Fortunately, we found that the maximum light absorption occurs in the range of very small filling factors. When $\mathrm{p}=350 \mathrm{~nm}$ and $\mathrm{FF}=0.2$, the maximum light absorption occurs, which is about $99.99973 \%$, and the wavelength is $274.3 \mathrm{~nm}$. This wavelength was adopted as the working wavelength for QE calculations in subsequent studies. In the range of the selected period, no matter what filling factor is selected, the maximum light absorption of the NHA can reach more than $80 \%$.

Fig. 3 (b) and (c) respectively show the dependence of the absorption spectrum on the period and filling factor in the wavelength range of $200 \mathrm{~nm}-400 \mathrm{~nm}$. The structure where the largest light absorption occurs does not exhibit the best light absorption on the overall wavelength. The light absorption of NHA at $\mathrm{p}=250 \mathrm{~nm}$ shows the best light absorption in the wavelength range of $250 \mathrm{~nm}-350 \mathrm{~nm}$. As the filling factor decreases, the absorption shows an increasing trend, which not only provides the possibility of enhanced light absorption, but also reduces the utilization of GaN materials. In addition, the results show that the largest light absorption of the NHA is approximately $20 \%$ higher than that of the thin film material. 

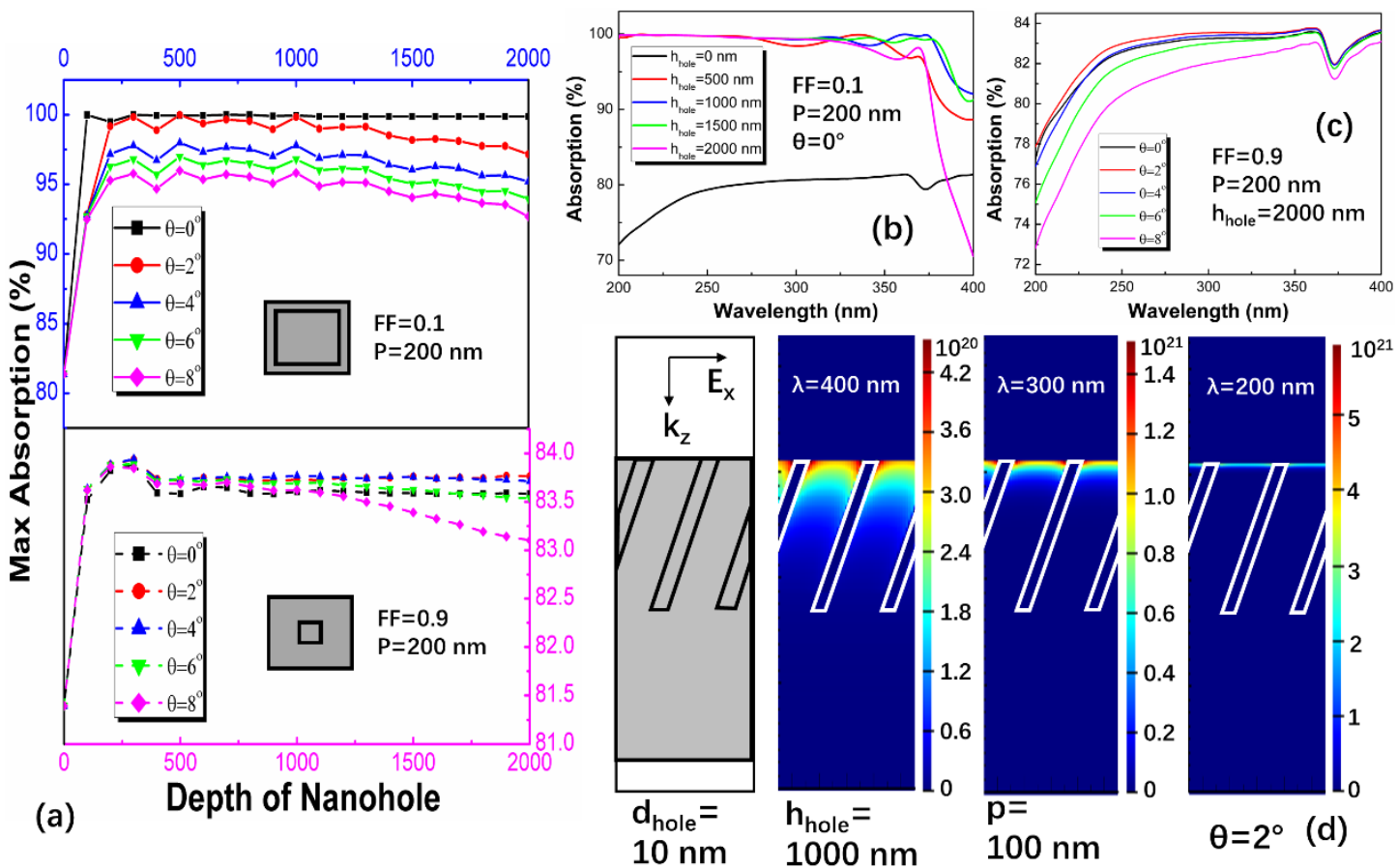

Figure 4. (a) The average absorption of inclined GaN NHA with different inclined angle as a function of hole depth, at filling factors of 0.1 and 0.9.(b) When the $F F=0.1, p=200 \mathrm{~nm}$, the dependence of the absorption spectrum of the vertical NHA on the hole depth. (c) When $p=200$ $\mathrm{nm}, \mathrm{FF}=0.9$, and the $\mathrm{h}_{\text {hole }}=2000 \mathrm{~nm}$, the dependence of the absorption spectrum of the NHA on the inclined angle. (d) Schematic diagram of inclined nanohole structure, photogeneration rate distribution, $\lambda=400 \mathrm{~nm}, \lambda=300 \mathrm{~nm}$, and $\lambda=200 \mathrm{~nm}$.

Fig. 4(a) shows the dependence of the average absorption on the inclined angle and hole depth of the GaN NHA with filling factors of 0.1 and 0.9 , respectively, and the period is fixed at $200 \mathrm{~nm}$. It can be observed that when the filling factor is 0.1 , the average absorption of the NHA is already very high, and the increase in the inclined angle has only a negative contribution to the average absorption. The difference is that a smaller inclined angle positively improves the light absorption of the NHA with a large filling factor, especially in the case of a deeper hole. However, when the inclined angle is increased to $8^{\circ}$, the light absorption drops significantly. In addition, we found that when the hole depth is about $400 \mathrm{~nm}$, the average light absorption has reached the highest, and deeper holes do not lead to higher light absorption, which is consistent with Cheney's report [12].

Fig. 4 (b) and (c) depict the dependence of the inclined NHA on the hole depth and inclined angle, respectively. When the hole depth is $0 \mathrm{~nm}$, the array is a planar film structure. Compared with the planar film, the light reflection of the oblique and vertical 
nanohole structure is significantly reduced. This is because the sudden change in refractive index between air and the planar film causes large light reflections. For the nanohole structure, the effective refractive index is low, thereby providing a buffer layer between the air and the underlying GaN film. The depth of the $500 \mathrm{~nm}$ nanohole is sufficient to collect most of the ultraviolet light without the need for auxiliary surface reorganization with deeper holes. The NHA structure is mechanically stronger, therefore it is more popular than self-supporting nanowires that are fragile and easy to break. In addition, the proposed NHA structure is interconnected, which providing excellent carrier transport within the structure as opposed to isolated nanowire structures. The enhancement of light absorption by the nanohole can be explained by the following theory. Light enters the nanohole, and then the light bounces back and forth between the walls of the nanohole. Each bounce will absorb part of the ultraviolet light, which will eventually lead to a large amount of absorption by the substrate. The increase in the effective optical path length leads to improved light collection. Inclination within a small angle can also increase the length of the effective optical path.

Fig. 4(d) shows a schematic diagram of the inclined GaN NHA and the photogeneration rate diagram on the $\mathrm{x}-\mathrm{z}$ plane, where the wavelength of incident light is $400 \mathrm{~nm}, 300 \mathrm{~nm}$, and $200 \mathrm{~nm}$, respectively. The inclined NHA can effectively trap sunlight into the structure, and a greatly enhanced photogeneration rate is observed under different sunlight wavelengths. When the wavelength of sunlight is $200 \mathrm{~nm}$, it is mainly enhanced at the top of the inclined NHA, indicating that sunlight has been absorbed before reaching the bottom of the structure. When the wavelength of sunlight increases to $400 \mathrm{~nm}$, the photogeneration rate at the top of the inclined nanohole is relatively weak, which indicates that lower light reflection and stronger electric field mode are observed in the inclined nanohole structure, and sunlight has been effectively coupled into the structure.

3.2 Quantum efficiency of GaN nanohole arrays 


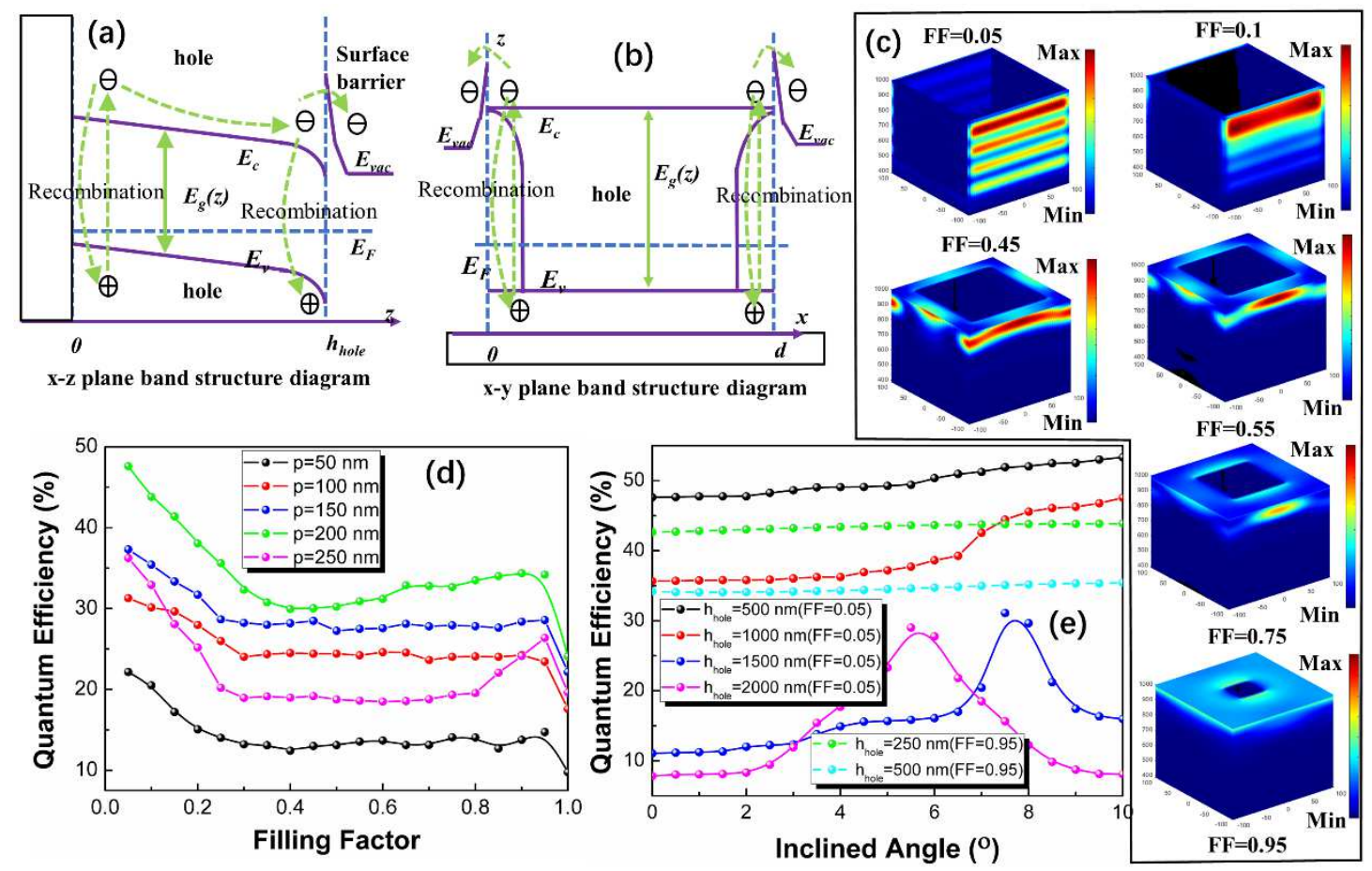

Figure 5. The band structure diagram of GaN NHA in the (a) x-z plane and (b) $x-y$ plane. $E_{C}$ is the bottom of the conduction band, $E_{V}$ is the top of the valence band, $E_{v a c}$ is the vacuum level, $E_{F}$ is the Fermi level, and $E_{\mathrm{g}}(\mathrm{z})$ is the band gap along the $\mathrm{z}$ axis. (c) Three-dimensional photogeneration rate of GaN NHA with different filling factors and a period of $50 \mathrm{~nm}$. (d) The QE of vertical GaN NHA with different period as a function of filling factor. (e) The QE of inclined GaN NHA with different hole depth as a function of inclined angle.

Fig. 5 (a) and (b) illustrate the simple band structure diagrams on the $\mathrm{x}-\mathrm{z}$ plane and $x-y$ plane, respectively. The electron movement process in the photocathode can be explained by the Spicer three-step model shown in Fig. 5 (a) and (b): the GaN NHA absorbs photons $\left(\mathrm{h} v>\mathrm{E}_{\mathrm{g}}\right)$ to generate electron-hole pairs, which transition from the bottom of the valence band to the top of the conduction band, and then move to the side and top surface of the nanohole through carrier diffusion and finally emit to the vacuum. The three-dimensional photogeneration rate of a GaN NHA with a period of $50 \mathrm{~nm}$ is shown in Fig. 5 (c). Herein, only photogeneration rate of NHA with a few representative filling factors are shown. We can be surprised to find that as the filling factor increases, the concentration of the photogeneration rate distribution of the NHA is continuously reduced. The photogeneration rate changes of NHA with periods of $100 \mathrm{~nm}, 150 \mathrm{~nm}$, $200 \mathrm{~nm}$, and $250 \mathrm{~nm}$ all show this trend, which is shown in Fig. S1-S5 of the supplementary material.

In addition, the results of Fig. S1-S5 show that the GaN NHA with a period of 200 
$\mathrm{nm}$ and a filling factor of 0.05 exhibit the most excellent photogeneration rate characteristics. Therefore, the NHA with this structural parameter obtains the highest QE, which is about 47.6\%, as shown in Fig. 5(d). At a small period, the characteristic size of the nanohole structure is much smaller than that of longer wavelength light, and it may not produce a strong scattering effect. Longer wavelengths will easily reflect or penetrate the nanohole structure, resulting in low QE. As period increases, the optimized QE will increase because the size of the nanohole gets closer and closer to the center of the ultraviolet spectrum. When period continues to increase, the distance between adjacent hole becomes larger and larger, resulting in high light reflection and transmission. When the filling factor is less than 0.3 , the $\mathrm{QE}$ decreases due to insufficient light absorption as the filling factor increases. When the filling factor is greater than 0.8 , the area of the top surface of the NHA also increase as the filling factor increases. However, most of the photogenerated carriers are located near the top surface of the NHA, then the increase in carriers emitted from the top surface is greater than the lack of carriers due to reduced absorption, resulting in a small increase in QE.

The photogeneration rate of GaN NHA with different hole depths is plotted in Fig. S6. It can be observed that as the hole depth increases, the resonance fringes on the side of the NHA become denser, but the overall photogeneration rate shows a downward trend. As shown in Fig. 5(e), similar to the relationship between enhanced light absorption and hole depth, the QE decrease as the hole depth increases. However, the dependence of the GaN NHA on the inclined angle is more profound. When the hole depth is $1500 \mathrm{~nm}$ and $2000 \mathrm{~nm}$ in the NHA, the QE show a peak in the array. The peak appears when the inclined angle is $\arctan \left(p / h_{\text {hole }}\right)$, and the lower right corner of the nanohole is at the boundary of the period. In the array with deep and small holes, although the inclined angle does not contribute enough to light absorption, the QE still increases as the inclined angle increases. This is because the inclined nanoholes are more conducive to the electron emission from the side, which can also be observed from Fig. 2(a). At a inclined angle of $10^{\circ}$, the GaN NHA with a filling factor of 0.05 and a hole depth of $500 \mathrm{~nm}$ can achieve the QE of 53.3\%. Since the electron collection side is located on the top of the nanohole, electrons emitted from the side of the 
nanohole are easily shielded and cannot reach the collection end on the top of the cathode. Therefore, it is still necessary to fully consider the collection probability of electrons.

\subsection{Collection efficiency of GaN nanohole array}
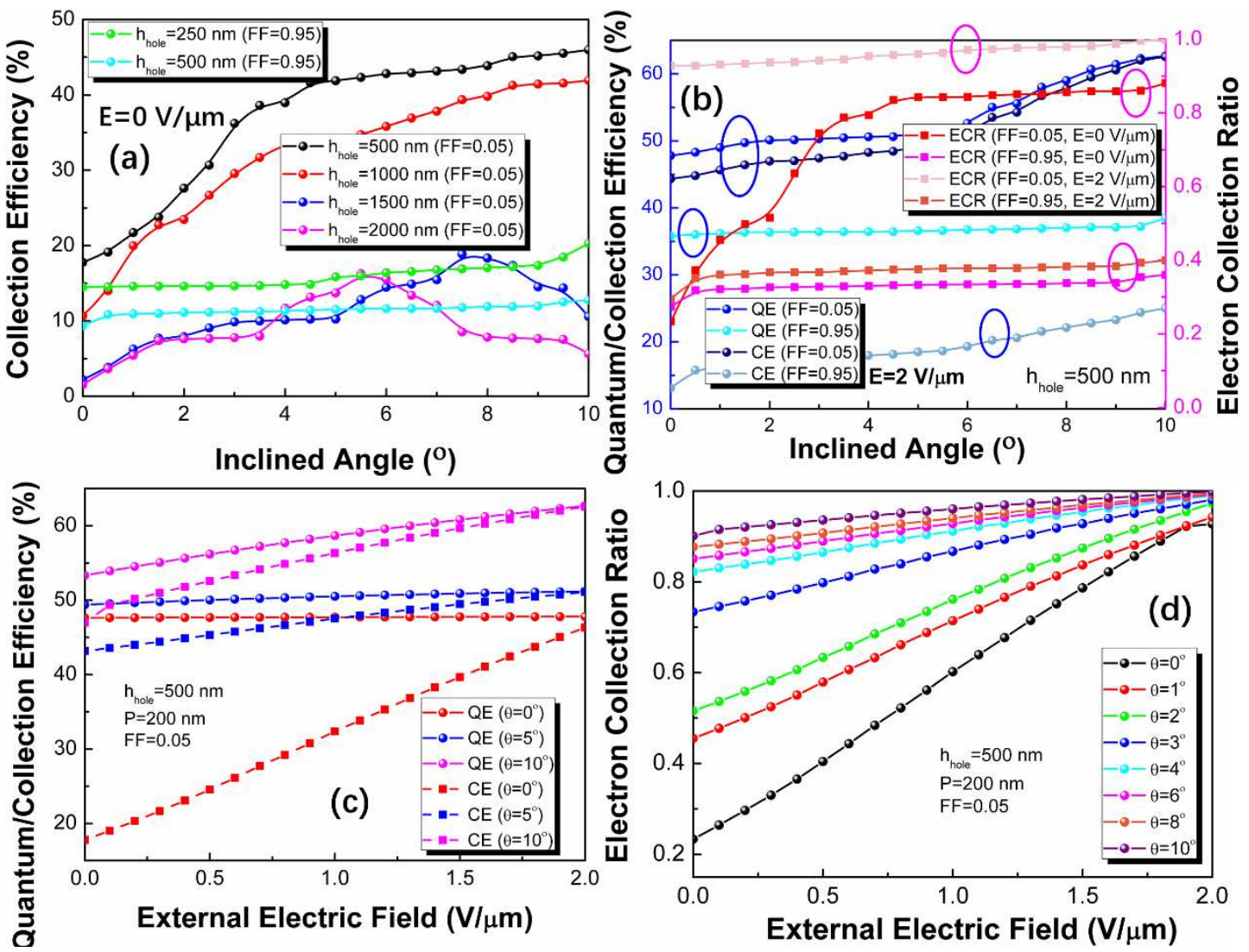

Figure 6. (a) The CE of inclined GaN NHA with different hole depths as a function of inclined angle, at the filling factors of 0.05 and 0.95 . (b) The $\mathrm{QE} / \mathrm{CE}$ and the electron collection ratio of the

field-assisted inclined GaN NHA with field intensity of $0 \mathrm{~V} / \mu \mathrm{m}$ and $2 \mathrm{~V} / \mu \mathrm{m}$ as a function of inclined angle. (c) The QE and CE of inclined GaN NHA with inclined angles of $0^{\circ}, 5^{\circ}$, and $10^{\circ}$ under different external electric field intensity. (d) The electron collection ratio of GaN NHA with different inclined angles as a function of external electric field intensity.

The calculation of QE only includes the emission of electrons from the surface of the nanohole. However, most of the electrons emitted from the sides are absorbed by the surface again, making the actual current in the device far less than ideal. According to Eq. (10)-(17), the CE of the inclined GaN NHA is calculated as shown in Fig. 6(a). The change trend of the $\mathrm{CE}$ of the GaN NHA still mainly follows the change trend of the QE. When the hole depth is $1500 \mathrm{~nm}$ and $2000 \mathrm{~nm}$, the CE of NHA also has a peak. In addition, it can be found that the inclined angle has a significant effect on improving the CE. For example, the CE of a NHA with a hole depth of $500 \mathrm{~nm}$ and a filling factor 
of 0.05 is only $17.8 \%$ when the inclined angle is $0^{\circ}$, but it can reach $46 \%$ when the inclined angle is $10^{\circ}$. This is mainly because a part of the side of the nanohole has a very large collection probability in the larger inclined angle of the array.

The positive effect of the external electric field can be found from our previous work $[24,25]$. The external electric field can deflect the direction of emission of electrons that have escaped from the side of the nanowire, and the electric field force accelerates the movement of the electrons to the collecting side to ensure as much collection as possible. The QE and CE of NHA with an external field intensity of 2 $\mathrm{V} / \mu \mathrm{m}$ as a function of the hole depth and inclined angle are plotted in Fig. S7 of the supplementary material. Both the QE and the CE of the NHA increased with the introduction of the external electric field, and the highest values rose to $62.7 \%$ and $62.6 \%$ respectively. In other words, with the assistance of a $2 \mathrm{~V} / \mu \mathrm{m}$ field intensity, the collection ratio of electrons is almost as high as $100 \%$, which can also be observed from Fig. 6(b). The blue curve plots the $\mathrm{QE}$ and $\mathrm{CE}$ of an array with filling factors of 0.05 and 0.95 , and a hole depth of $500 \mathrm{~nm}$ as a function of inclined angle. The pink curve plots their electron collection ratio. The electron collection ratio is the ratio between the electrons collected and the electrons emitted. The electron collection ratio of the fieldassisted NHA cathode with a small filling factor (large diameter of hole) is between $0.88-0.998$, and the degree of electron collection is very high. However, even with the aid of a large field intensity, the electrons emitted from the bottom and side surfaces of the nanohole with a large filling factor (small diameter of hole) cannot obtain a good collection effect.

In order to observe the effects of the external electric field more intuitively, we describe the relationship between the $\mathrm{QE} / \mathrm{CE}$, the electron collection ratio and field intensity in Fig. 6(c) and (d), respectively. The external field intensity improves the CE of the vertical NHA most significantly. As the inclined angle of the nanohole and the external field intensity gradually increase, the collection ratio of electrons is also steadily increasing. When the external field intensity reaches $2 \mathrm{~V} / \mu \mathrm{m}$, almost all electron from the NHA can be collected regardless of the inclined angle. Therefore, the field-assisted method may be very necessary for the vacuum-type nanostructured 
photocathode. In subsequent research, we will have to consider introducing fieldassisted devices into the photocathode for photocurrent testing.

3.4 Graded compositional AlGaN nanohole array
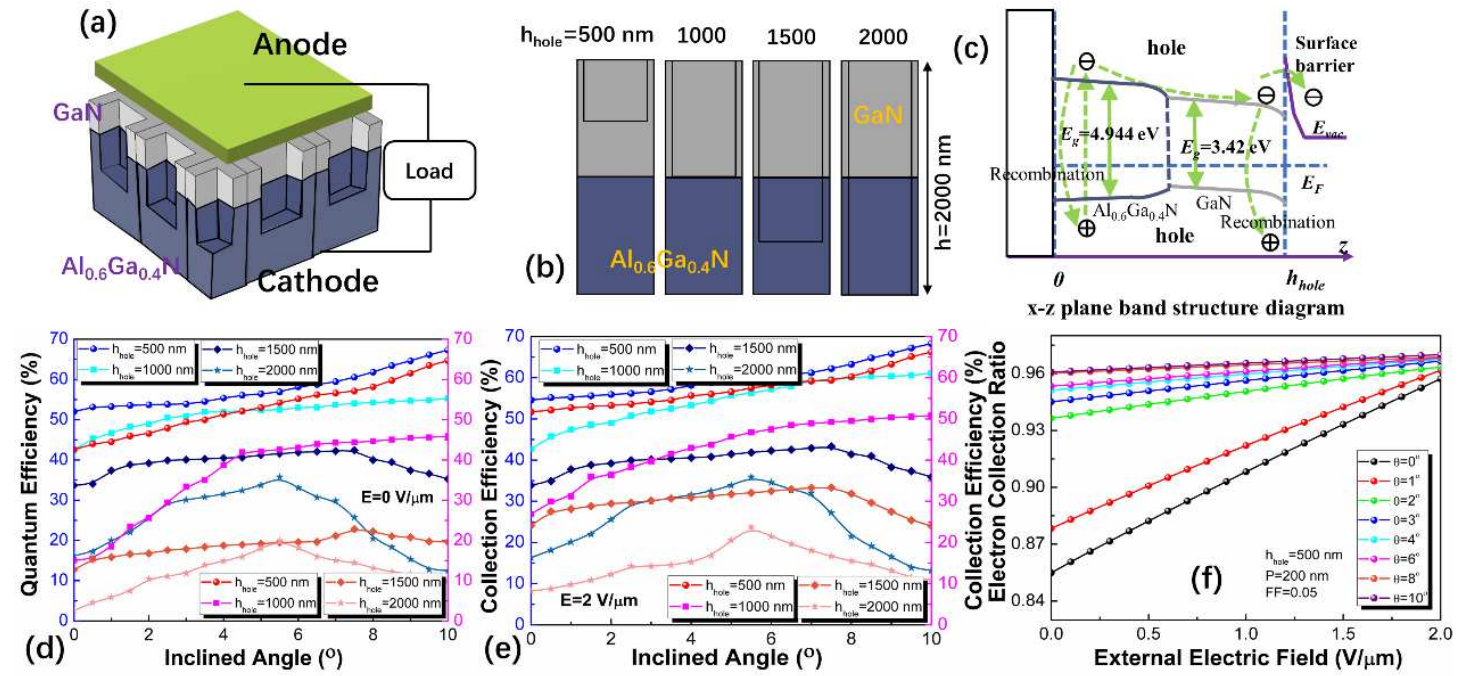

Figure 7. (a) A schematic diagram of the device model of the field-assisted graded compositional AlGaN NHA. (b) Side view of graded compositional AlGaN nanohole with different hole depths.

(c) Schematic diagram of the band structure of graded compositional AlGaN NHA in the $\mathrm{x}-\mathrm{z}$ plane. (d) The QE and CE of the inclined AlGaN NHA with different hole depth at a field intensity of $0 \mathrm{~V} / \mu \mathrm{m}$ as a function of inclined angle. (e) The QE and CE of the inclined AlGaN NHA with different hole depth at a field intensity of $2 \mathrm{~V} / \mu \mathrm{m}$ as a function of inclined angle. (f) The electron collection ratio of the inclined AlGaN NHA with different inclined angle as a function of field intensity.

The built-in electric field can be introduced by means of graded composition. Under the pull of the built-in electric field, some of the electrons originally from the side will drift to the top surface for emission, which is conducive to the emission and collection of electrons. The field-assisted graded compositional AlGaN NHA device is shown in Fig. 7(a), and the dark blue part represents $\mathrm{Al}_{0.6} \mathrm{Ga}_{0.4} \mathrm{~N}$ material. When the NHA has a graded composition structure, the hole depth determines the properties of the material exposed on the side and bottom of the nanohole. As shown in Fig. 7(b), holes with a depth of $500 \mathrm{~nm}$ only exposure to $\mathrm{GaN}$ materials, and $\mathrm{Al}_{0.6} \mathrm{Ga}_{0.4} \mathrm{~N}$ materials are exposed on the bottom and sides of holes with a depth of $1500 \mathrm{~nm}$. The hierarchical electric field generated between different sublayers will tilt the band structure of the sublayer, and this change is non-continuous, as shown in Fig. 7(c). The $\mathrm{Al}_{0.6} \mathrm{Ga}_{0.4} \mathrm{~N}$ layer and the $\mathrm{GaN}$ emitting layer form a heterojunction, which acts as an electron mirror for the photoelectrons generated in the effective layer near the interface, which is 
beneficial to improve the QE of the photocathode.

Fig. 7 (d) and (e) respectively depict the QE and CE of the AlGaN NHA with different hole depths at field intensity of $0 \mathrm{~V} / \mu \mathrm{m}$ and $2 \mathrm{~V} / \mu \mathrm{m}$ as a function of inclined angle. The blue curve represents the QE, and the pink curve represents the CE. When the inclined angle is $10^{\circ}$, the $\mathrm{QE}$ and $\mathrm{CE}$ of the graded compositional AlGaN NHA are increased to $67.29 \%$ and $64.65 \%$, respectively. Compared with a uniform GaN NHA, the efficiency is increased by 1.4 times. With the assistance of $2 \mathrm{~V} / \mu \mathrm{m}$ field intensity, the $\mathrm{QE}$ and $\mathrm{CE}$ continue to increase to $68.28 \%$ and $66.23 \%$. Because the built-in electric field have a part of contributions, the positive influence of the external electric field on the graded compositional AlGaN NHA becomes smaller. This can also be observed from the relationship between the electron collection ratio and the electric field in Fig. 7(f). However, in a field intensity of $2 \mathrm{~V} / \mu \mathrm{m}$, the vertical uniform GaN NHA has an electron collection ratio of 0.4 , which rises to 0.96 for the $\mathrm{AlGaN}$ array with a graded composition, which is of decisive significance for the vertical NHA.

\section{Conclusion}

In summary, this work can be used to provide design guidelines for the preparation of inclined GaN NHA photocathodes. A systematic study was carried out using FDTD and three-dimensional continuity equations to illustrate the effects of period, filling factor, hole depth and inclined angle on the light trapping ability and photoemission performance of $\mathrm{GaN}$ and $\mathrm{AlGaN}$ NHA. The alterations in period, filling factor, hole depth and inclined angle provide additional parameters for changing the morphology of the nanohole. Compared with planar film, NHA have significantly enhanced light absorption. The GaN NHA with a period of $200 \mathrm{~nm}$ and a filling factor of 0.05 exhibits excellent photogeneration rate. The $\mathrm{QE}$ of the array with an inclined angle of $10^{\circ}$ is about $47.6 \%$, and the $\mathrm{CE}$ is $46 \%$. When the field intensity of $2 \mathrm{~V} / \mu \mathrm{m}$ is superimposed, the QE and CE of the array are 62.7 and 62.6\%, respectively. The field-assisted method has profound significance for the electron collection of the NHA. In addition, the graded compositional $\mathrm{AlGaN}$ structure has a more improved effect on the vertical NHA, and the electron collection ratio is increased by 2.4 times compared with the uniform $\mathrm{GaN}$ NHA. Therefore, the NHA structure provides a potential solution for the GaN 
photocathode industry to improve cell efficiency.

Funding: This study was funded by Qing Lan Project of Jiangsu Province-China (grant number 2017-AD41779) and the Six Talent Peaks Project in Jiangsu Province-China (grant number 2015-XCL-008).

\section{Conflict of interest statement}

The authors declare that they have no conflict of interests in either personal or financial aspects.

\section{Reference}

[1] Wang XH, Chang BK, Du YJ, Qiao JL. Quantum efficiency of GaN photocathode under different illumination. Applied Physics Letter, 2011, 99:042102.

[2] Qiao JL, Chang BK, Qian YS, Du XQ, Zhang YJ, Gao P, Wang XH, Guo XY, Niu J, Gao YT. Study of spectral response characteristics of negative electron affinity GaN photocathode. Acta Physica Sinica, 2010,59,3577-3582.

[3] Treu J, Xu X, Ott K, Sailer K, Abstreiter G, Finley JJ, Koblmuller G. Optical absorption of composition-tunable InGaAs nanowire arrays. Nanotechnology, 2019,30,495703.

[4] Lu FF, Liu L, Tian J. Residual gas adsorption effect on the stability of Cs-activated GaN nanowire photocathode. Applied Surface Science, 2019, 497: UNSP 143791.

[5] Xia SH, Liu L, Kong Y, Diao Y. Study of Cs adsorption on (100) surface of [001]-oriented GaN nanowires: A first principle research. Applied Surface Science, 2016, 387:1110-1115.

[6] Xu ZP, Huangfu HC, He L, Wang JZ, Yang D, Guo JW, Wang HY. Light-trapping properties of the $\mathrm{Si}$ inclined nanowire arrays. Optics Communications, 2017, 382:332-336.

[7] Xu ZP, Huangfu HC, Li XW, Qiao HL, Guo WC, Guo JW, Wang HY. Role of nanocone and nanohemisphere arrays in improving light trapping of thin film solar cells. Optics Communications, 2016, 377:104-109.

[8] Wei WR, Tsai ML, Ho ST, Tai SH, Ho CR, Tsai SH, Liu CW, Chung RJ, He JH. Above-11\%Efficiency Organic-inorganic hybrid solar cells with omnidirectional harvesting characteristics by employing hierarchical photon-trapping structures. Nano Letters, 2013, 13(8): 3658-3663.

[9] Makableh YF, Al-Fandi M, Khasawneh M, Tavares CJ. Comprehensive design analysis of ZnO anti-reflection nanostructures for Si solar cells. Superlattices and Microstructures, 2018, 124:1-9.

[10] Han SE, Chen G. Optical Absorption Enhancement in Silicon Nanohole Arrays for Solar Photovoltaics. Nano Letters, 2010, 10(3): 1012-1015.

[11] Du QG, Kam CH, Demir HV, Yu HY, Sun XW. Enhanced optical absorption in nanopatterned silicon thin films with a nano-cone-hole structure for photovoltaic applications. Optics Letters, 2011, 36(9): 1713-1715.

[12] Chen TG, Yu PC, Chen SW, Chang FY, Huang BY, Cheng YC, Hsiao JC, Li CK, Wu YR. 
Characteristics of large-scale nanohole arrays for thin-silicon photovoltaics. Progress in Photovoltaics, 2014, 22(4): 452-461.

[13] Hong L, Rusli, Wang XC, Zheng HY, Wang H, Yu HY. Design Guidelines for Si(111) Inclined Nanohole Arrays in Thin-Film Solar Cells. IEEE Transaction on Nanotechnology, 2014, 13(3): 431436.

[14] Deng C, Tan XY, Jiang LH, Tu YT, Ye M, Yi YS. Efficient light trapping in silicon inclined nanohole arrays for photovoltaic applications. Optics Communications, 2018, 407:199-203.

[15] Peng KQ, Wang X, Li L, Wu XL, Lee ST. High-Performance Silicon Nanohole Solar Cells. Journal of the American Chemical Society, 2010, 132(20): 6872-+.

[16] Zhang C, Li XF, Shang AX, Wu SL, Zhan YH, Yang ZH. Design of dual-diameter nanoholes for efficient solar-light harvesting. Nanoscale Research Letters, 2014, 9:481.

[17] Ding W, Xia DY, Li Q, Huang YP, Zheng M, Zhang LZ, Wang J, Zhang Y, Guo MF, Liu S, Su XL, Yun F, Hou X. Photonic crystal based on anti-reflection structure for $\mathrm{GaN} / \mathrm{InGaN}$ heterojunction solar cells. Proceedings of the SPIE. International Conference on Photonics and Optical Engineering, 2015, 9449:94491L.

[18] Han L, Zhao HP. Surface antireflection properties of GaN nanostructures with various effective refractive index profiles. Optics Express, 2014, 22:031907.

[19] Winnerl J, Kraut M, Hudeczek R, Stutzmann M. GaN nanowire arrays for photocatalytic applications II: influence of a dielectric shell and liquid environments. Applied Physics B, 2019, 125:77.

[20] Zou JJ, Chang BK. Gradient-doping negative electron affinity GaAs photocathodes. Optical Engineering, 2006, 45(5): 054001.

[21] Fu XQ, Ai YB. Quantum efficiency dependence on built-in electric fields in exponential-doped and graded-doped gallium arsenide photocathodes. Optik, 2012, 123(20): 1888-1890.

[22] Yang MZ, Guo J, Fu XQ, Liu ZH. Quantum efficiency of heterostructured AlN/AlxGa1-xN photocathodes with graded bandgap emission layer. Journal of Materials Science-Materials in Electronics, 2018, 29(14):12443-12450.

[23] Lu FF, Liu L, Tian J. Comparison of quantum and collection efficiency of field-assisted uniform-doping and exponential-doping GaN nanowire cathodes. International Journal of Energy Research, 2020, 44(3): 1751-1760.

[24] Liu L, Lu FF, Xia SH, Diao Y, Tian J. Improved electron capture capability of field-assisted exponential-doping GaN nanowire array photocathode. Journal of Materials Science and Technology, 2020, 42: 54-62.

[25] Liu L, Xia SH, Diao Y, Lu FF, Tian J. Enhancement of photoemission capability and electron collection efficiency of field-assisted GaN nanowire array photocathode. Nanotechnology, 2020, 31:025201.

[26] Diao Y, Liu L, Xia SH. Photon-enhanced thermionic emission solar energy converters with GaAs wire array cathode under external electric field. Applied Nanoscience, 2020, 10(3): 807-817.

[27] Guo HM, Wen L, Li XH, Zhao ZF, Wang YQ. Analysis of optical absorption in GaAs nanowire arrays. Nanoscale Research Letters, 2011, 6:617.

[28] Liu L, Diao Y, Xia SH. High-performance GaAs nanowire cathode for photon-enhanced thermionic emission solar converters. Journal Materials Science, 2019, 54:5605-5614.

[29] Zou J, Chang B, Chen H, Liu L. Variation of quantum-yield curves for GaAs photocathodes under illumination. Journal of Applied Physics. 2007, 101:033126. 
[30] Feng C, Zhang YJ, Qian YS, Chang BK, Shi F, Jiao GC, Zou JJ. Photoemission from advanced heterostructured $\mathrm{Al}_{\mathrm{x}} \mathrm{Ga}_{1-\mathrm{x}} \mathrm{As} / \mathrm{GaAs}$ photocathodes under multilevel built-in electric field. Optics Express, 2015, 23(15):19478-19488.

[31] Liu Y, Li QX, Wan LY, Kucukgok B, Ghafari E, Ferguson IT, Zhang X, Wang SC, Feng ZC, $\mathrm{Lu}$ N. Composition and temperature dependent optical properties of AlxGa1-xN alloy by spectroscopic ellipsometry. Applied Surface Science, 2017, 421(BSI): 389-396.

[32] Reddy P, Bryan I, Bryan Z, et.al. Charge neutrality levels, barrier heights, and band offsets at polar AlGaN. Applied Physics Letters, 2015, 107, 091603.

[33] Li J, Oder TN, Nakarmi ML, Lin JY, Jiang HX. Optical and electrical properties of Mg-doped p-type AlxGa1-xN. Applied Physics Letters, 2002, 80(7): 1210-1212.

[34] Cooper JW. Multiple corrections to the angular distribution of photoelectrons at low energies. Physical review A. 1990, 42(11): 6942-6945.

[35] Hanold KA, Garner MC, Continetti RE. Photoelectron-photofragment angular correlation and energy partitioning in dissociative photodetachment. Physical Review Letters. 1996, 77(16): 33358 .

[36] Fang X, Zhao CY, Bao H. Radiative behaviors of crystalline silicon nanowire and nanohole arrays for photovoltaic applications. Journal of Quantitative Spectroscopy and Radiative Transfer, 2014, 133: 579-588. 


\section{Supplementary material}

\section{Photo-absorption and electron collection of field-assisted GaN}

\section{nanohole array photocathode for solar cell applications}

Lei Liu*, Feifei Lu, Jian Tian, Xingyue Zhangyang, Zhisheng Lv

Department of optoelectronic technology, School of Electronic and Optical

Engineering, Nanjing University of Science and Technology, Nanjing 210094, China

Corresponding author: Lei Liu; E-mail:liu1133_cn@sina.com.cn

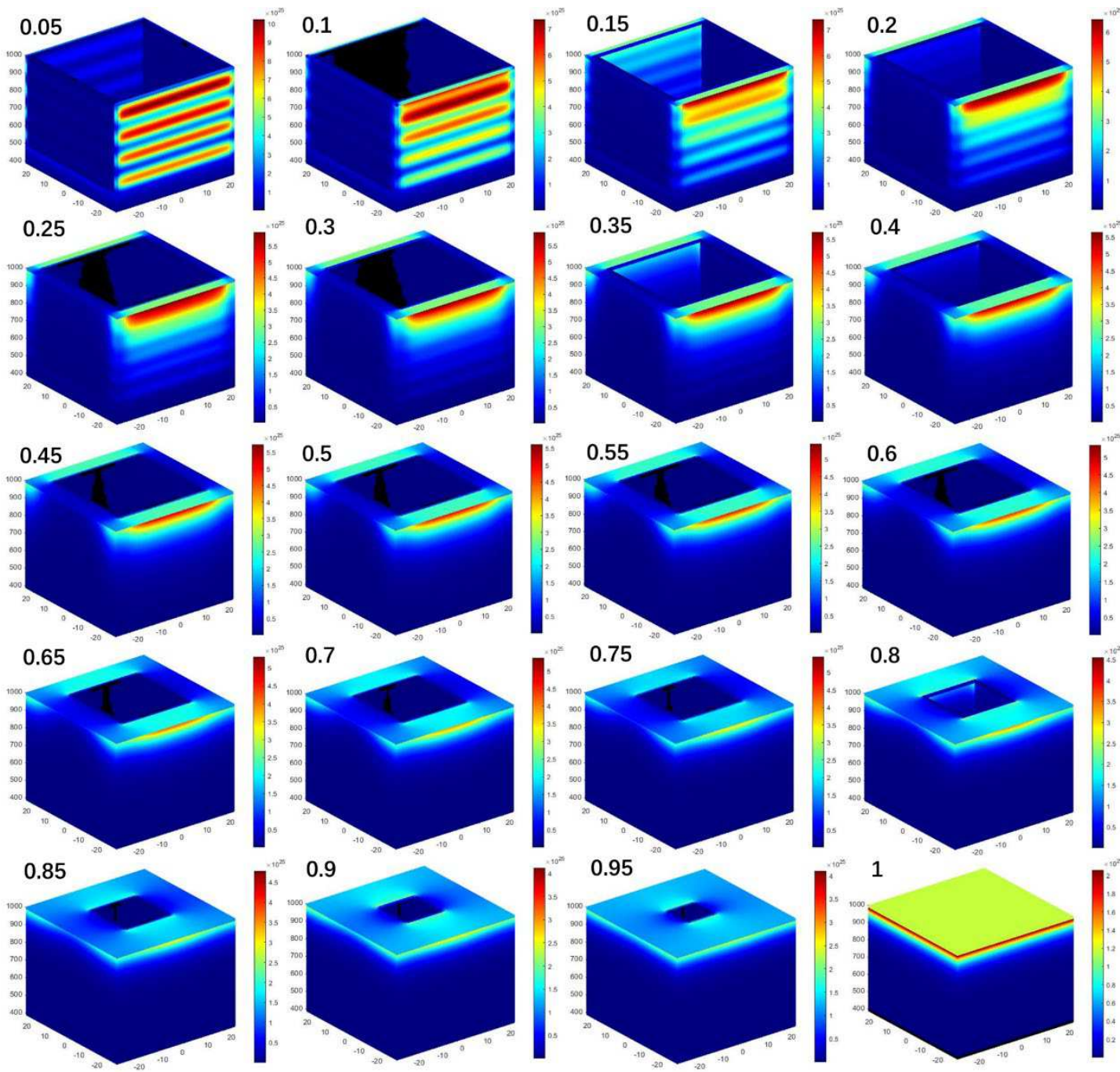

Figure S1. The photogeneration rate of a vertical GaN nanohole with a period of 50 $\mathrm{nm}$ and different filling factor. 

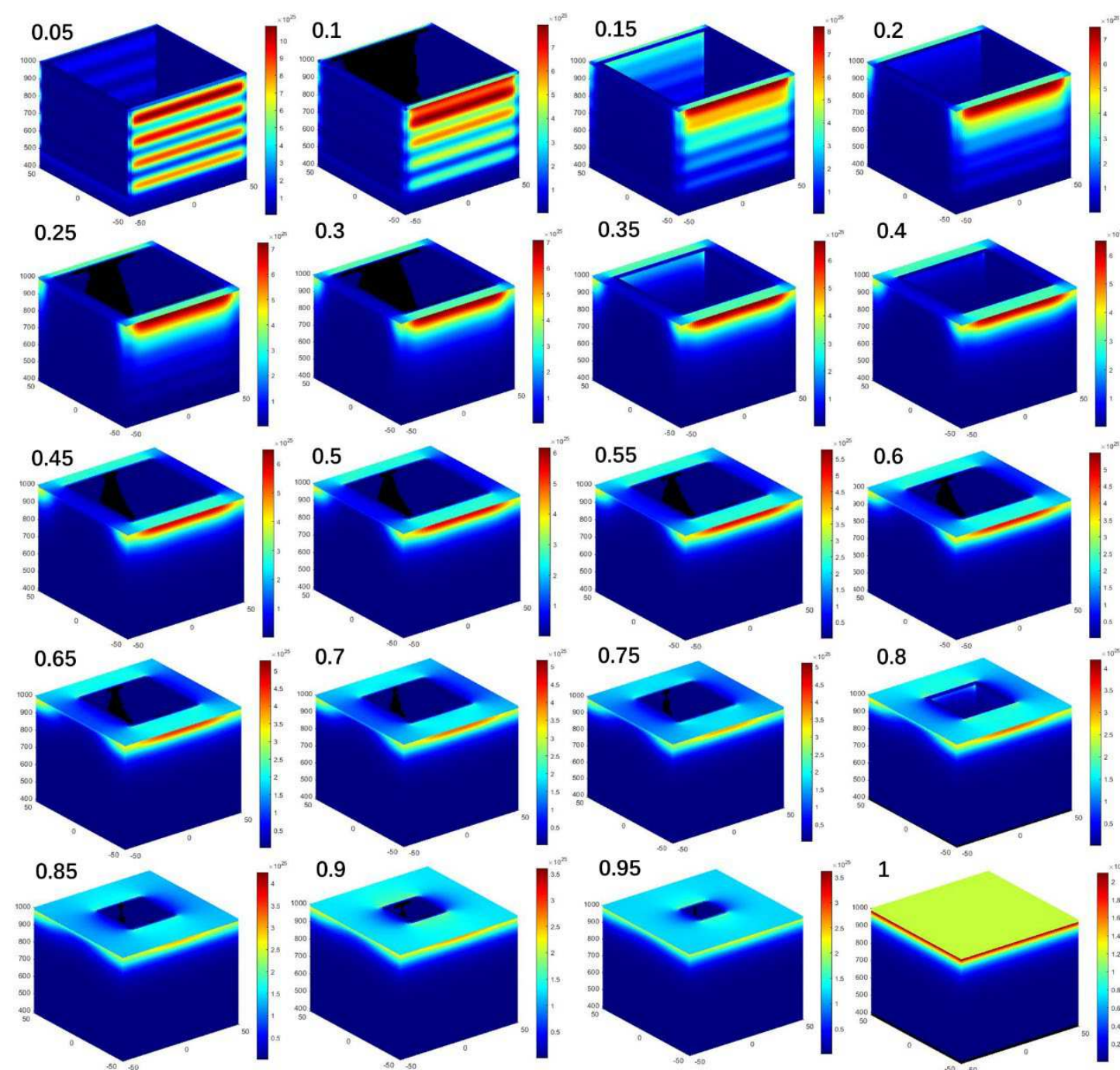

Figure S2. The photogeneration rate of a vertical GaN nanohole with a period of 100 $\mathrm{nm}$ and different filling factor. 

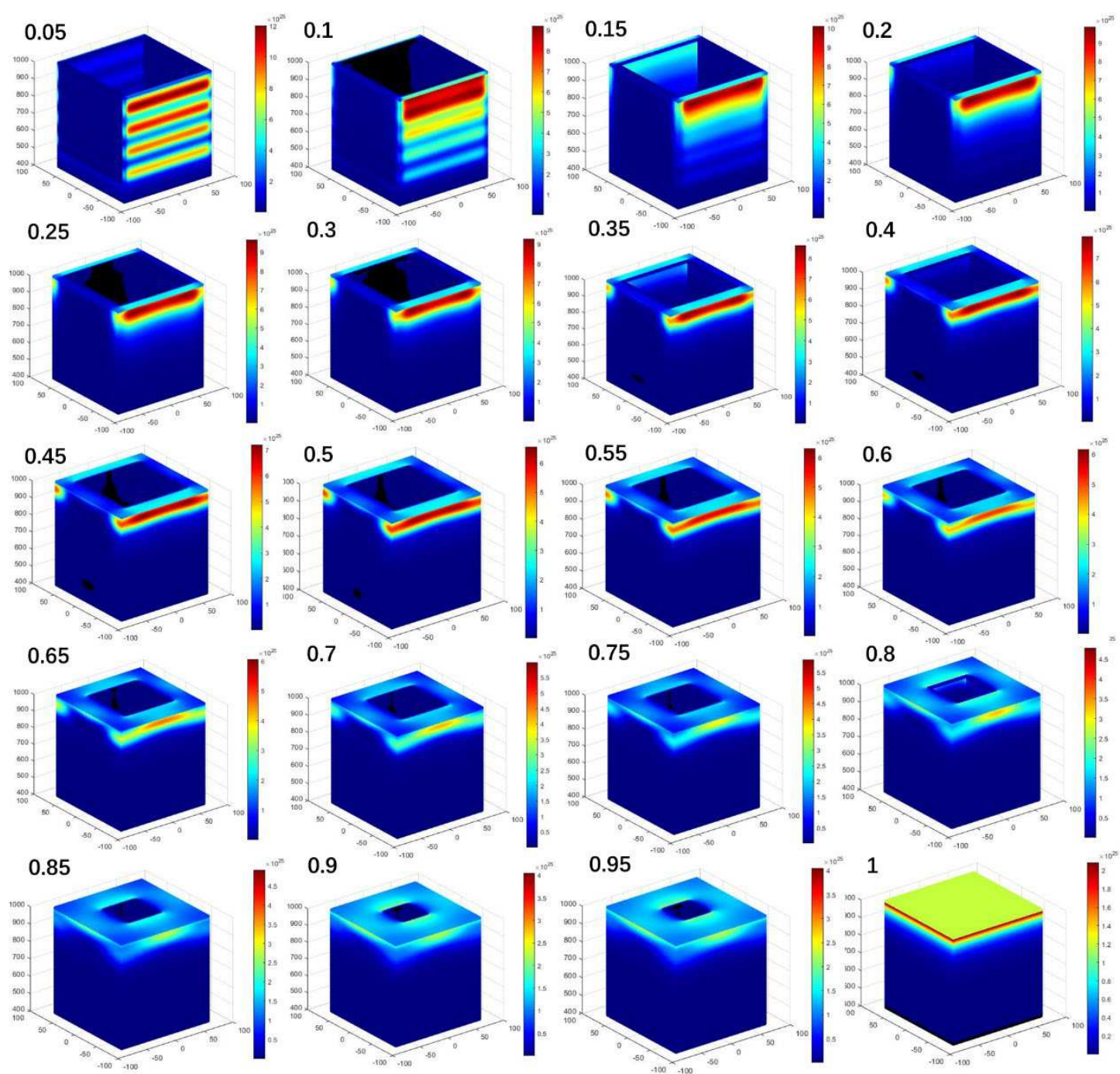

Figure S3. The photogeneration rate of a vertical GaN nanohole with a period of 150 $\mathrm{nm}$ and different filling factor. 

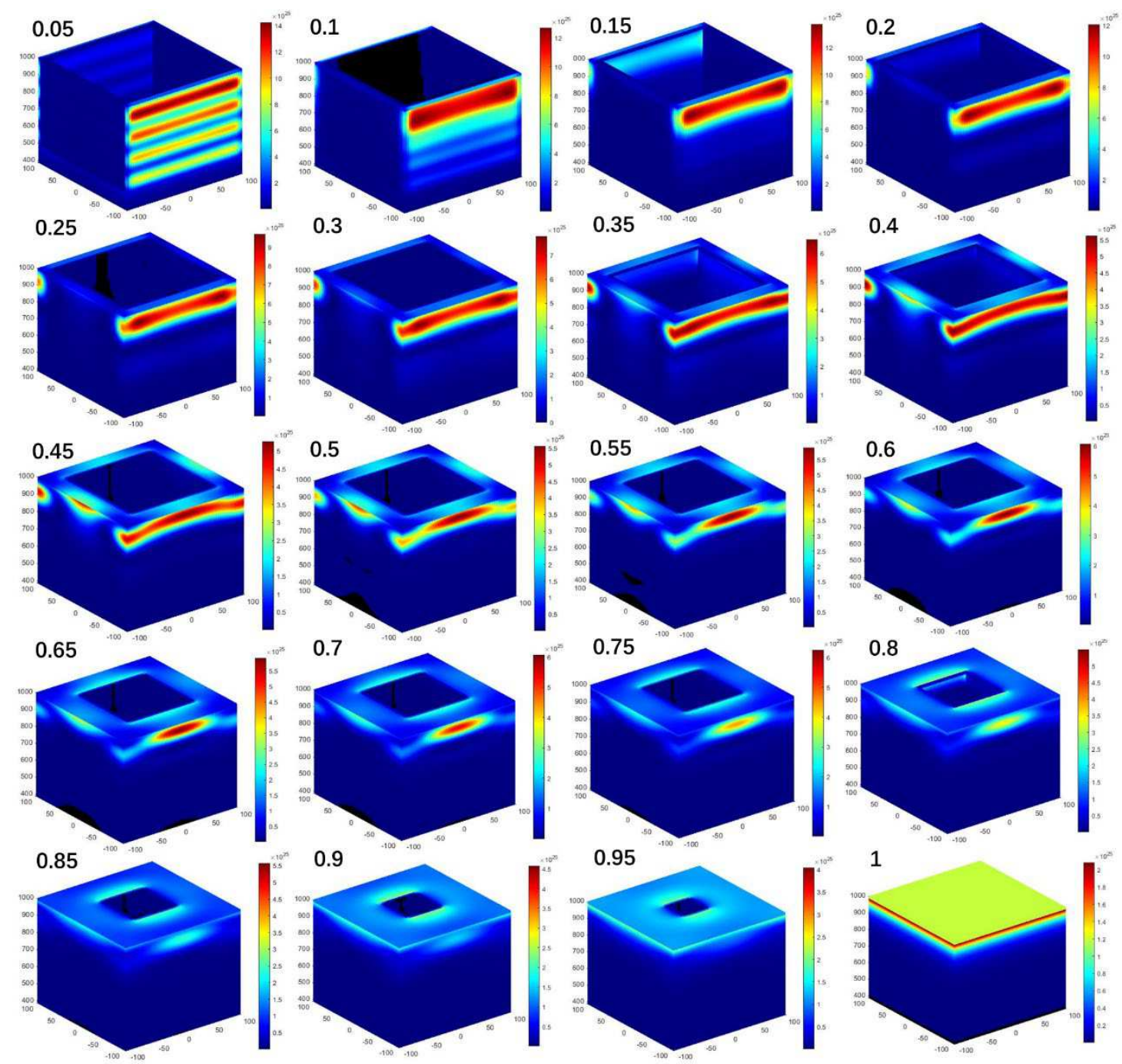

Figure S4. The photogeneration rate of a vertical GaN nanohole with a period of 200 $\mathrm{nm}$ and different filling factor. 


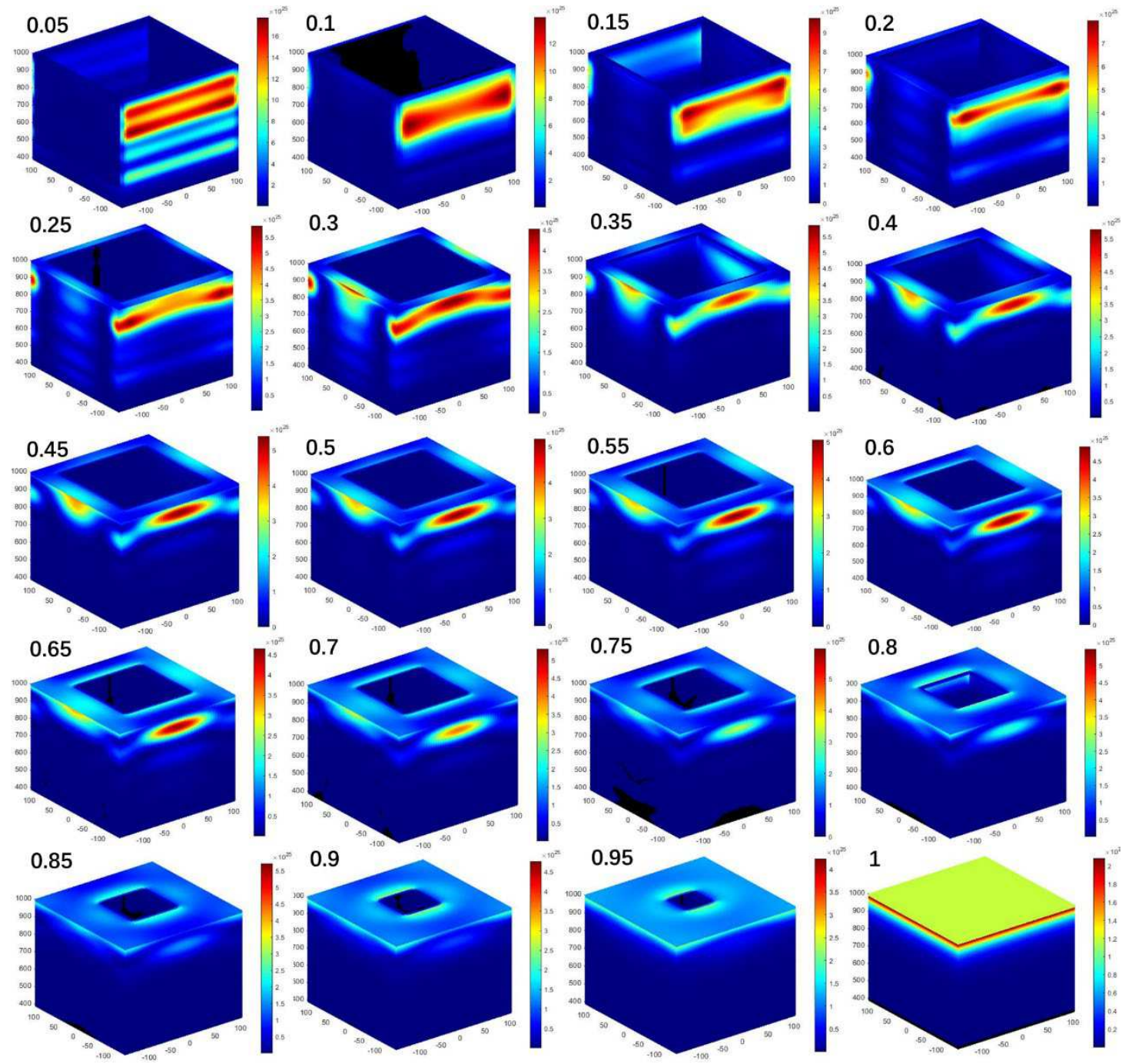

Figure S5. The photogeneration rate of a vertical GaN nanohole with a period of 250 $\mathrm{nm}$ and different filling factor.
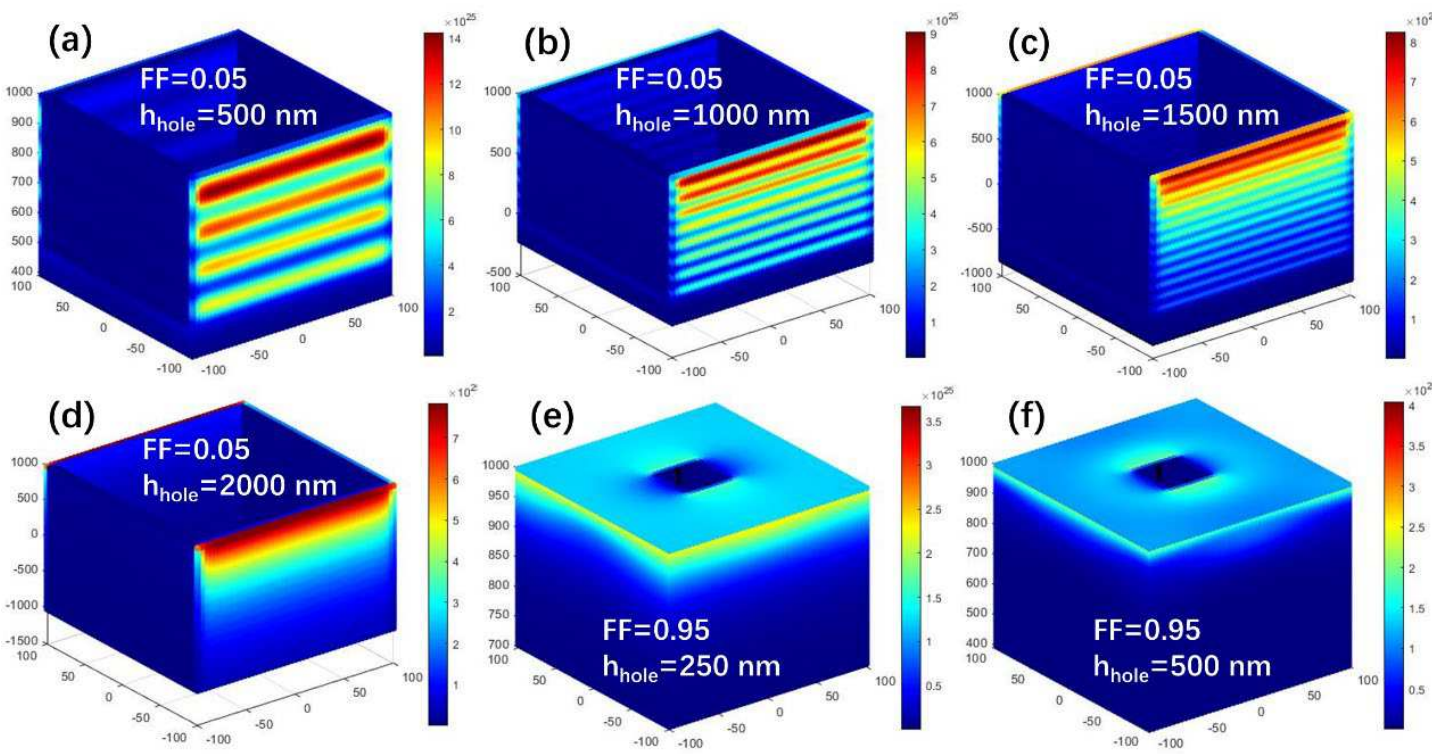

Figure S6. The photogeneration rate of a vertical GaN nanohole with a filling factor 
of 0.05 and different hole depth.
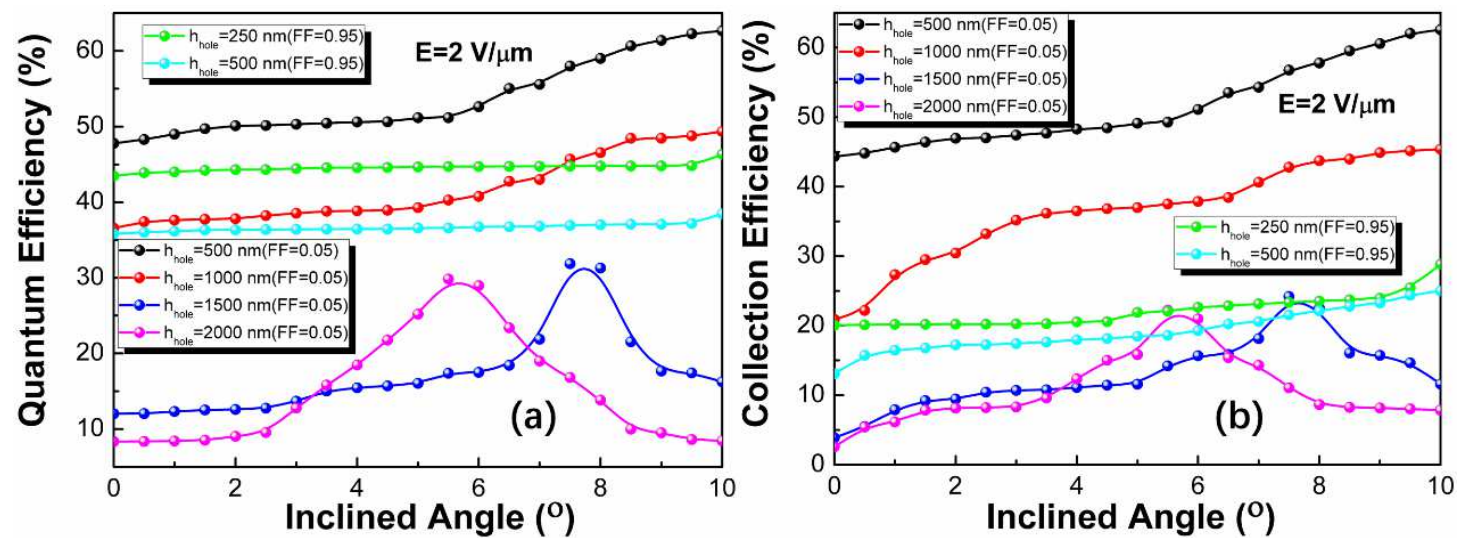

Figure S7. The (a) quantum efficiency and (b) collection efficiency of inclined GaN NHA with different hole depth as a function of inclined angle at a field intensity of 2 $\mathrm{V} / \mu \mathrm{m}$. 
Figures

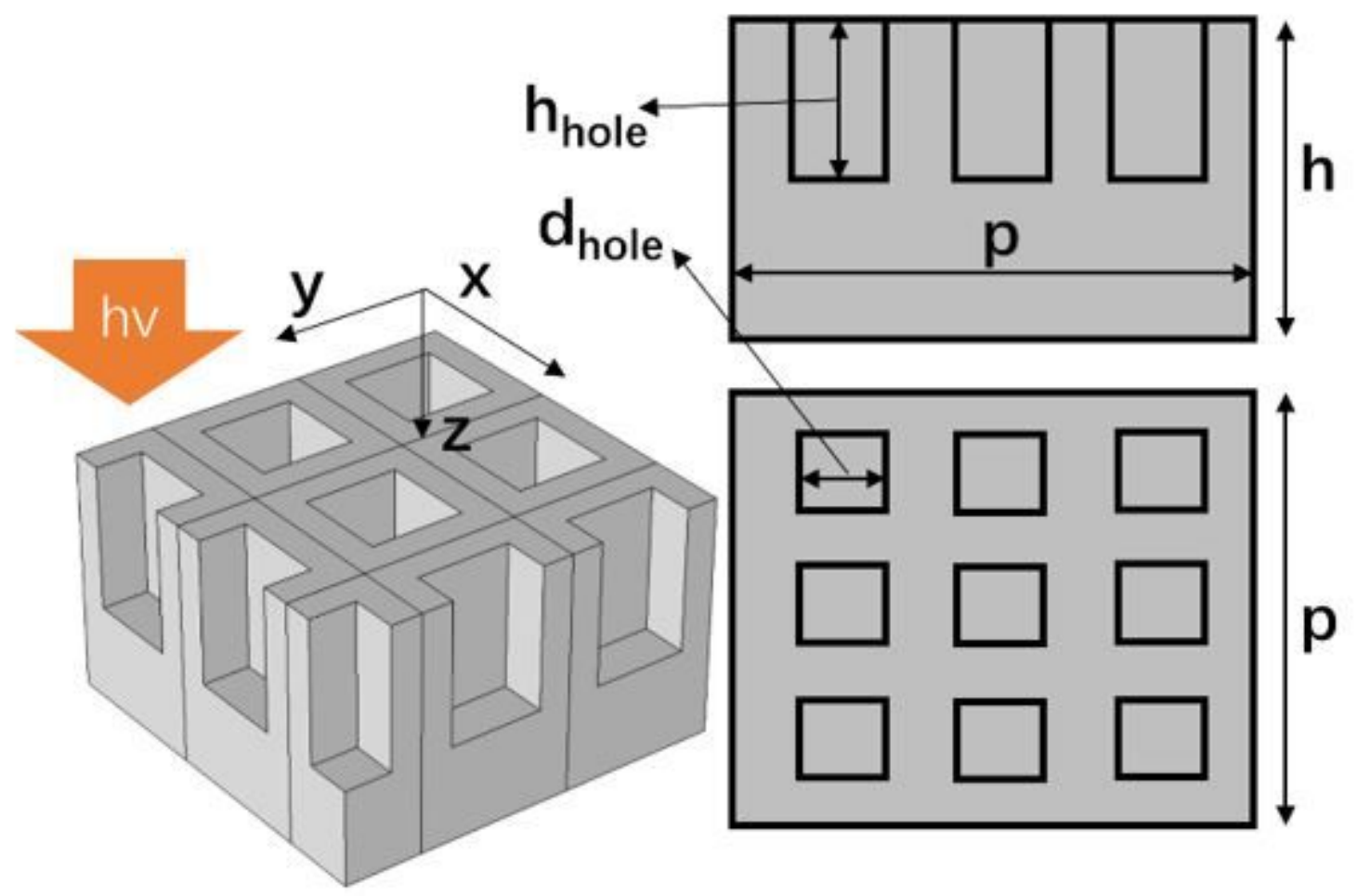

Figure 1

Structural model of GaN nanohole array. 

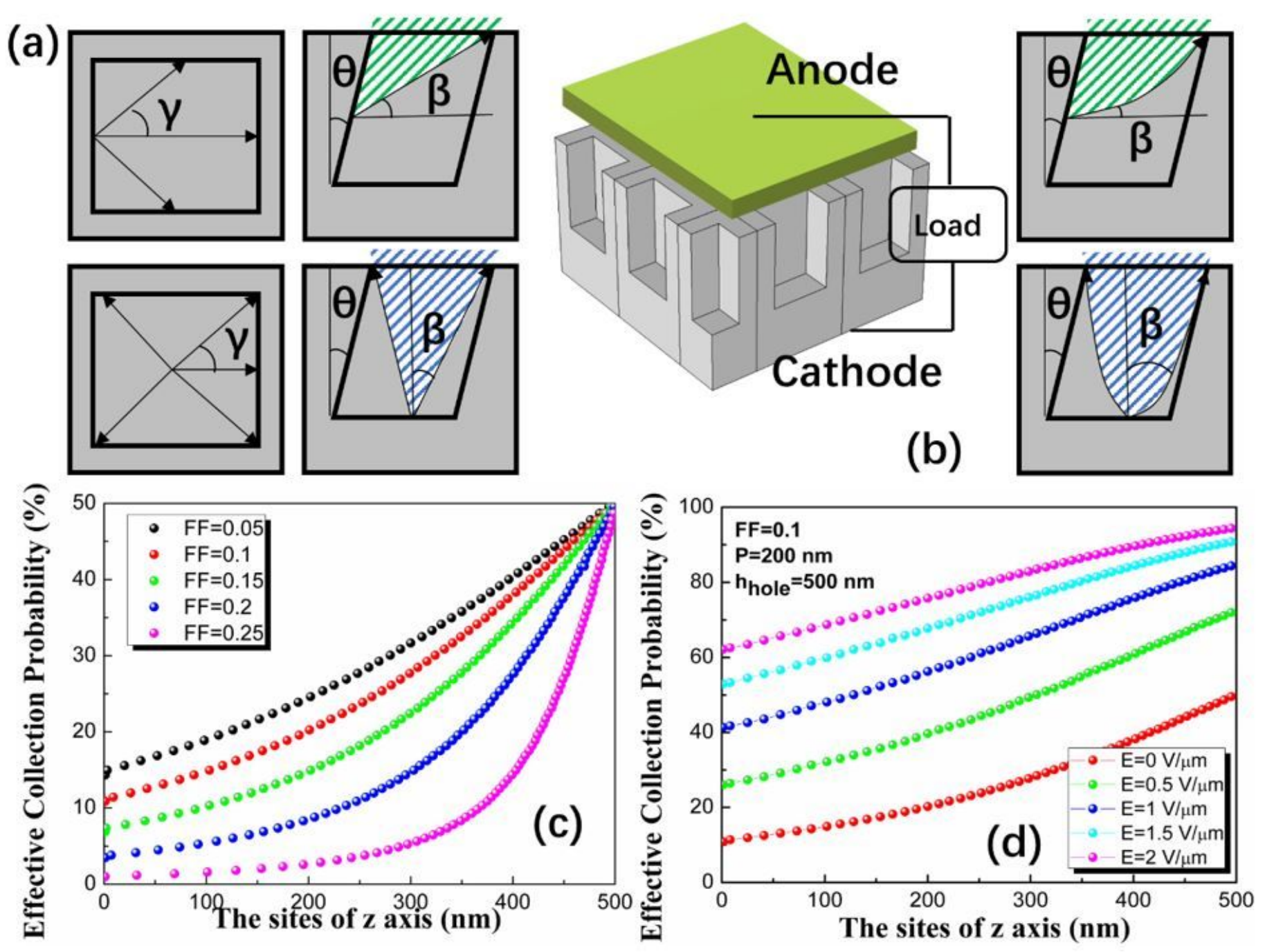

Figure 2

(a) The range where electrons can be collected from the inclined GaN NHA under no external electric field. (b) Left: A schematic diagram of the device model of the field-assisted GaN NHA. Right: The range where electrons can be collected from the inclined GaN NHA under an external electric field. (c) The dependence of the effective collection probability of electrons emitted from the side of the GaN NHA on the filling factor when there is no external electric field. (d) The dependence of the effective collection probability of electrons emitted from the side of the GaN NHA on the field intensity.
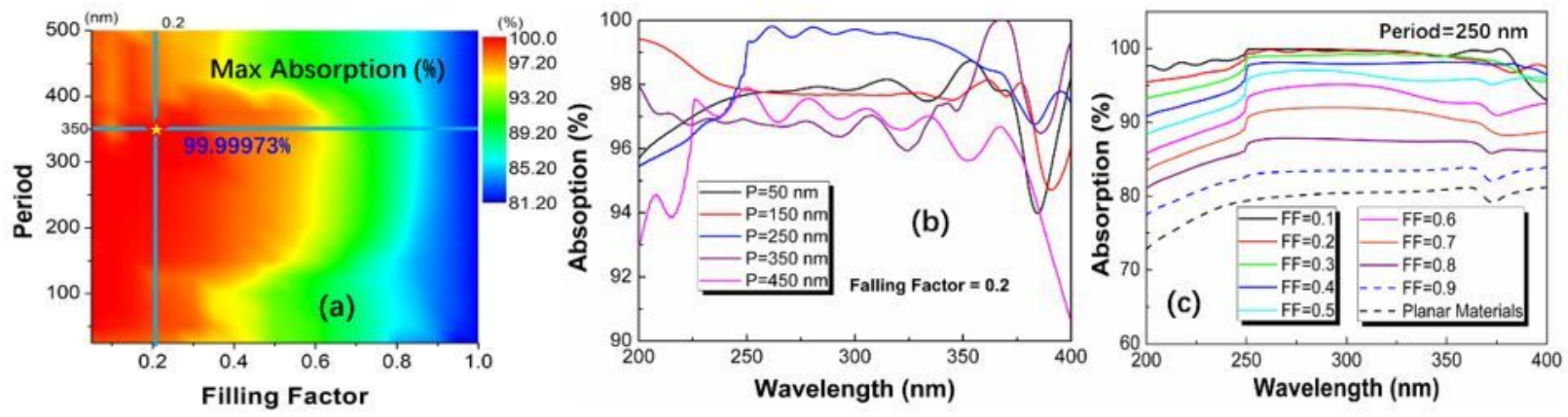


\section{Figure 3}

(a) Photo-absorption of a square vertical NHA as a function of period and filling factor. (b) When the filling factor is 0.2 , the dependence of the absorption spectrum of the vertical NHA on the period. (c) When the period is $250 \mathrm{~nm}$, the dependence of the absorption spectrum of the vertical NHA on the filling factor.

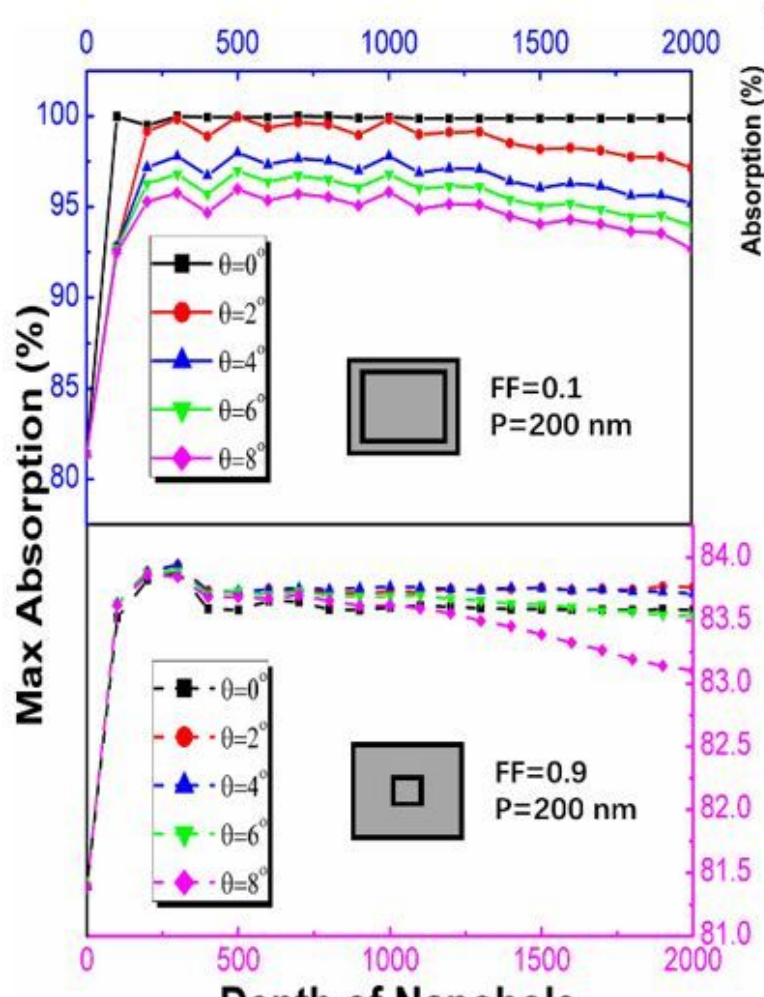

(a)

Depth of Nanohole
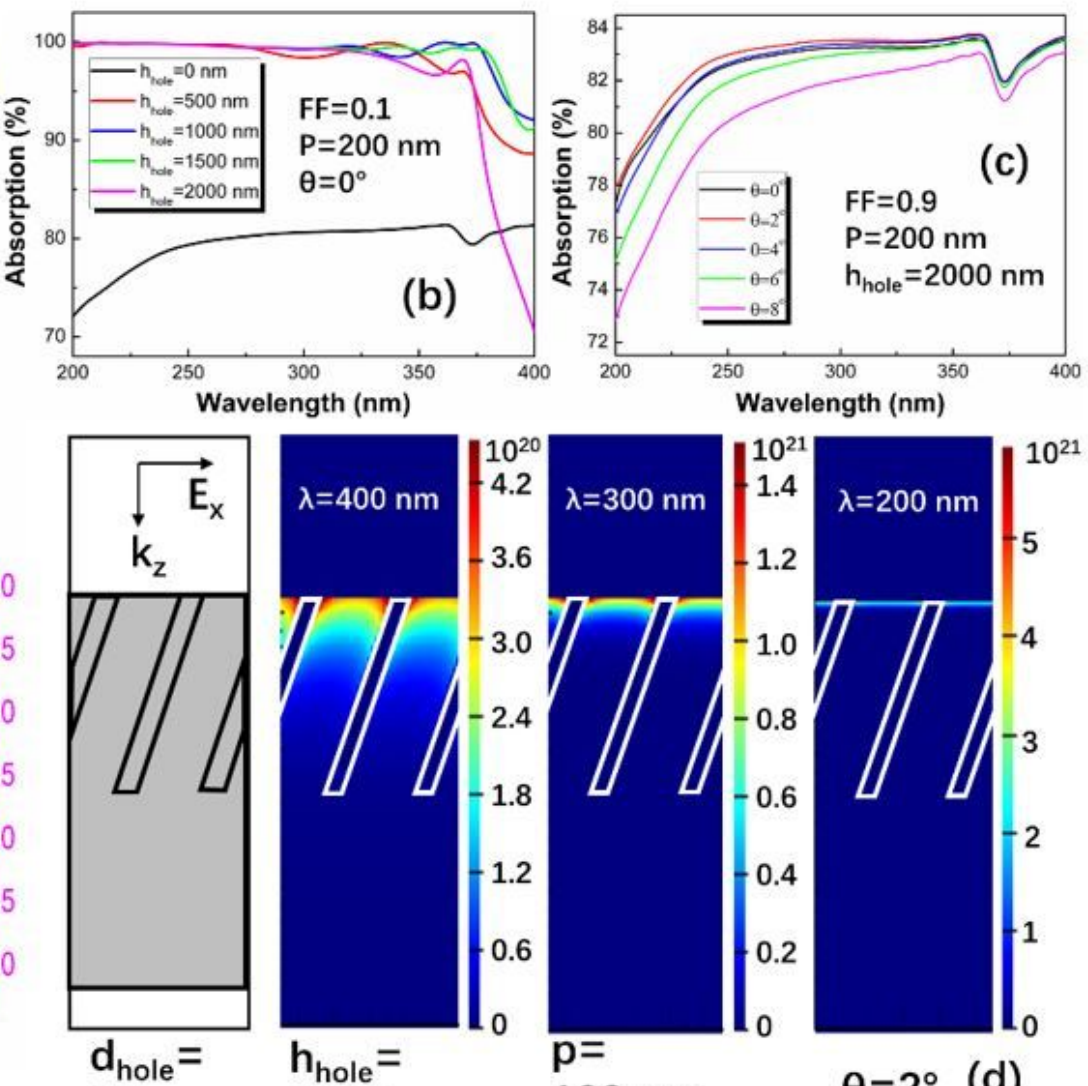

$10 \mathrm{~nm}$

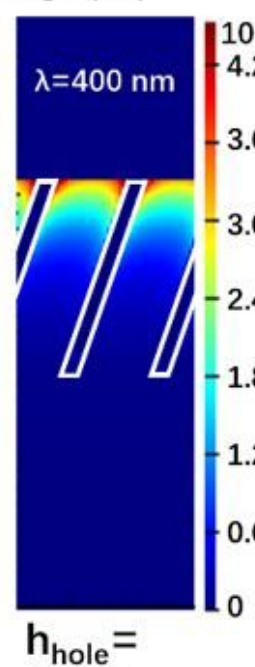

$1000 \mathrm{~nm}$

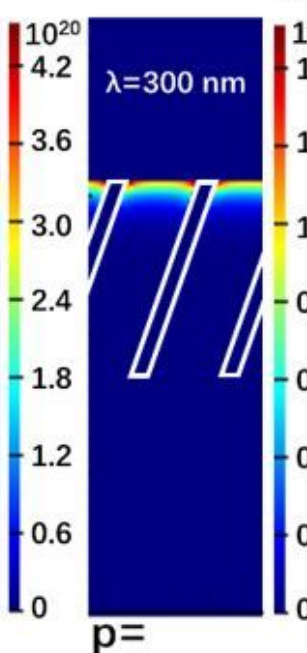

$100 \mathrm{~nm}$

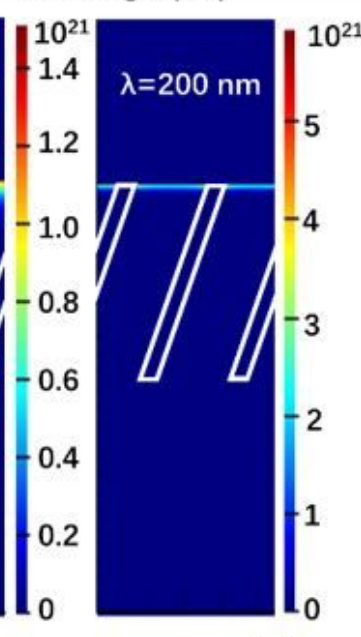

$\theta=2^{\circ}$ (d)

Figure 4

(a) The average absorption of inclined GaN NHA with different inclined angle as a function of hole depth, at filling factors of 0.1 and 0.9.(b) When the $F F=0.1, p=200 \mathrm{~nm}$, the dependence of the absorption spectrum of the vertical NHA on the hole depth. (c) When $p=200 \mathrm{~nm}, \mathrm{FF}=0.9$, and the hhole $=2000 \mathrm{~nm}$, the dependence of the absorption spectrum of the NHA on the inclined angle. (d) Schematic diagram of inclined nanohole structure, photogeneration rate distribution, $\lambda=400 \mathrm{~nm}, \lambda=300 \mathrm{~nm}$, and $\lambda=200 \mathrm{~nm}$. 


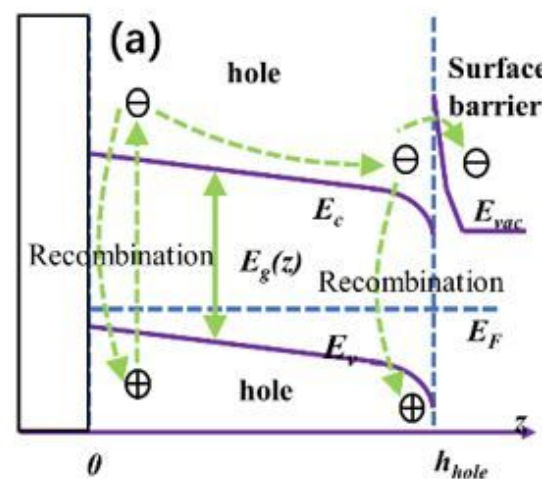

$\mathbf{x}-\mathbf{z}$ plane band structure diagram

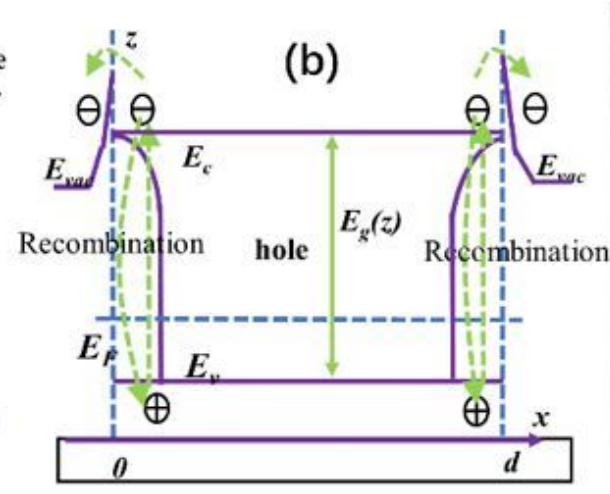

$x-y$ plane band structure diagram
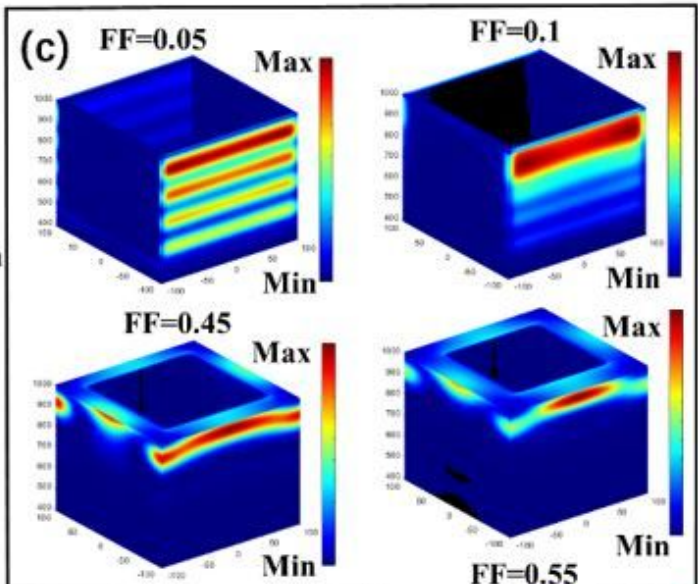

$\mathrm{FF}=\mathbf{0 . 5 5}$
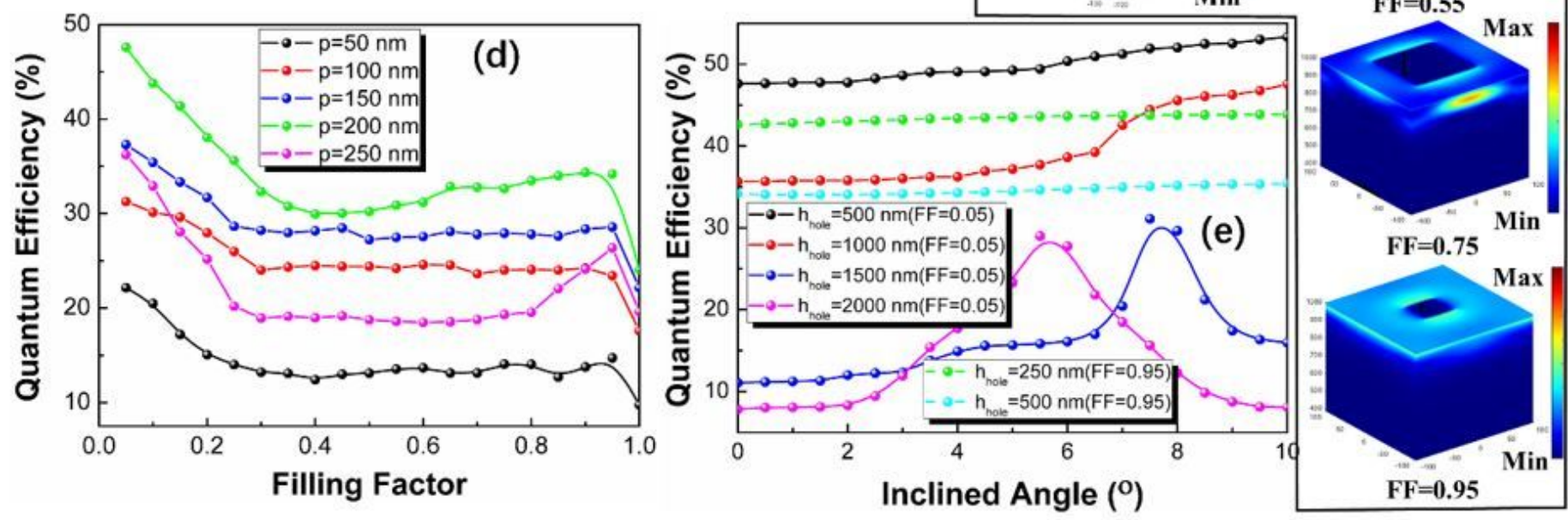

Figure 5

The band structure diagram of GaN NHA in the (a) $x-z$ plane and (b) $x-y$ plane. $\mathbb{\text { Q }}$ is the bottom of the

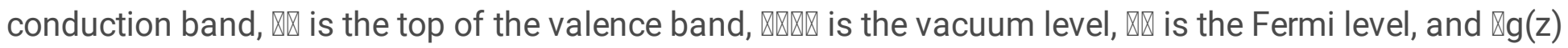
is the band gap along the $z$ axis. (c) Three-dimensional photogeneration rate of GaN NHA with different filling factors and a period of $50 \mathrm{~nm}$. (d) The QE of vertical GaN NHA with different period as a function of filling factor. (e) The QE of inclined GaN NHA with different hole depth as a function of inclined angle. 

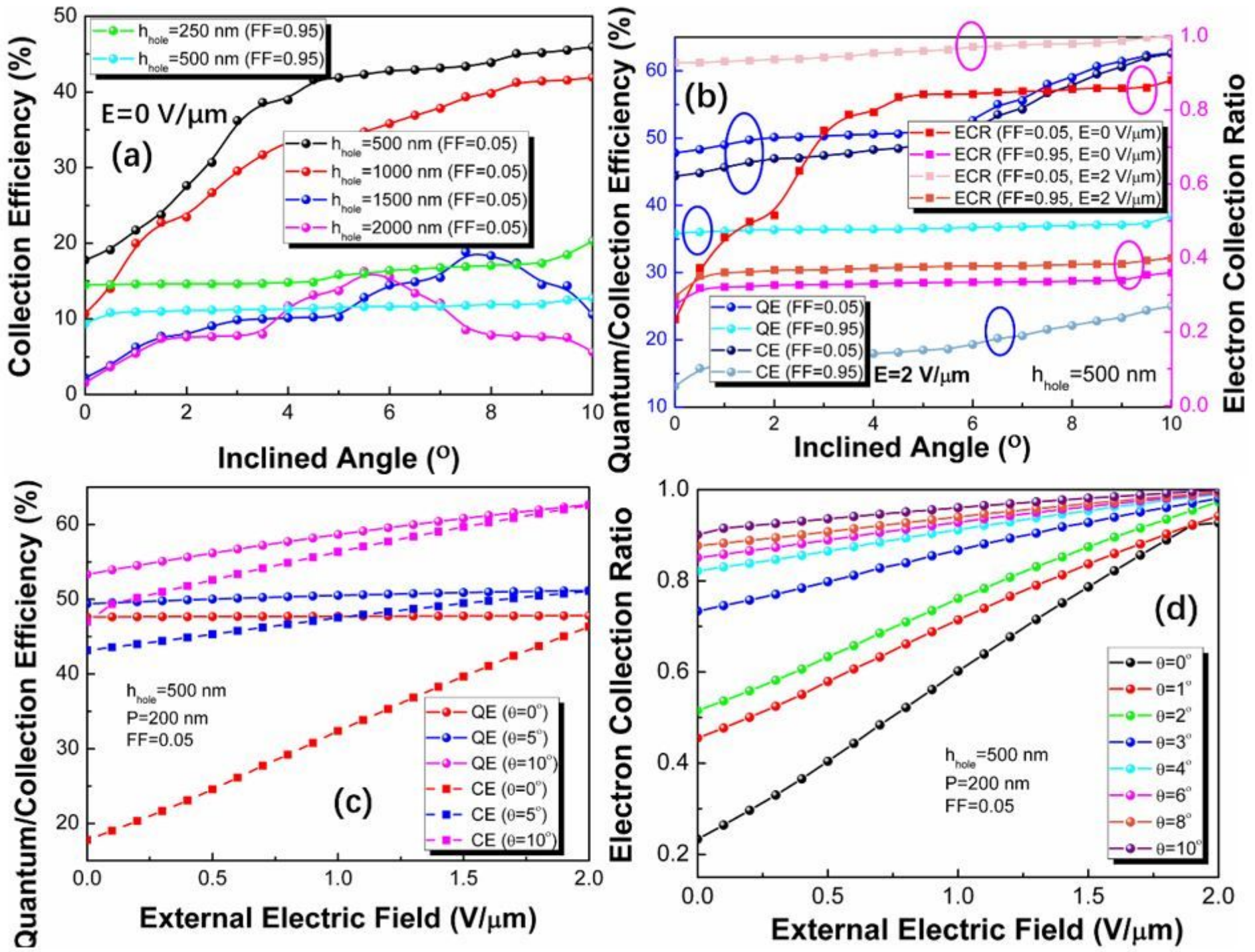

Figure 6

(a) The CE of inclined GaN NHA with different hole depths as a function of inclined angle, at the filling factors of 0.05 and 0.95 . (b) The QE/CE and the electron collection ratio of the field-assisted inclined GaN $\mathrm{NHA}$ with field intensity of $0 \mathrm{~V} / \mu \mathrm{m}$ and $2 \mathrm{~V} / \mu \mathrm{m}$ as a function of inclined angle. (c) The QE and CE of inclined GaN NHA with inclined angles of $0^{\circ}, 5^{\circ}$, and $10^{\circ}$ under different external electric field intensity. (d) The electron collection ratio of $\mathrm{GaN}$ NHA with different inclined angles as a function of external electric field intensity. 

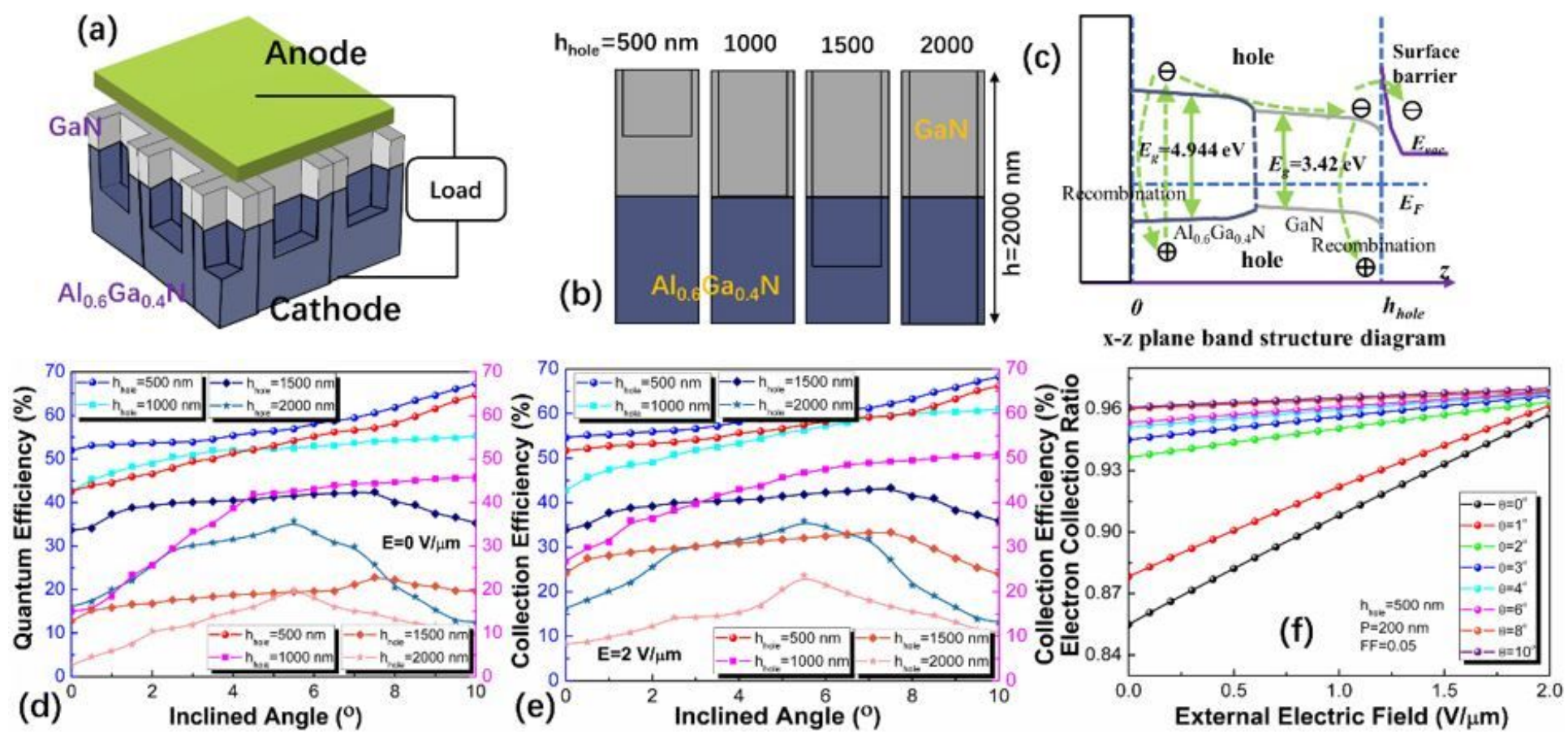

Figure 7

(a) A schematic diagram of the device model of the field-assisted graded compositional AIGaN NHA. (b) Side view of graded compositional AIGaN nanohole with different hole depths. (c) Schematic diagram of the band structure of graded compositional AIGaN NHA in the x-z plane. (d) The QE and CE of the inclined AlGaN NHA with different hole depth at a field intensity of $0 \mathrm{~V} / \mu \mathrm{m}$ as a function of inclined angle. (e) The QE and CE of the inclined AIGaN NHA with different hole depth at a field intensity of $2 \mathrm{~V} / \mu \mathrm{m}$ as a function of inclined angle. (f) The electron collection ratio of the inclined AIGaN NHA with different inclined angle as a function of field intensity. 